\title{
Vlasov gyrokinetic simulations of ion-temperature-gradient driven instabilities
}

G. Manfredi, M. Shoucri, R. O. Dendy, A. Ghizzo, and P. Bertrand

Citation: Physics of Plasmas 3, 202 (1996); doi: 10.1063/1.871846

View online: https://doi.org/10.1063/1.871846

View Table of Contents: http://aip.scitation.org/toc/php/3/1

Published by the American Institute of Physics

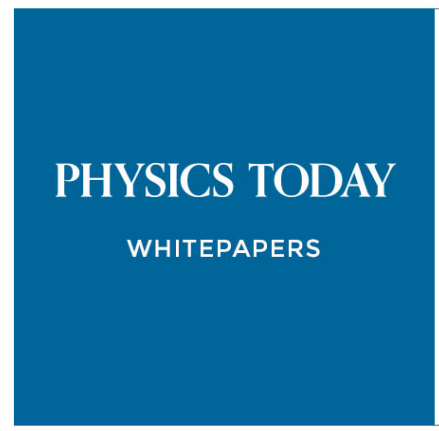

ADVANCES IN PRECISION MOTION CONTROL

Piezo Flexure Mechanisms and Air Bearings

\section{READ NOW}

PRESENTED BY PI 


\title{
Vlasov gyrokinetic simulations of ion-temperature-gradient driven instabilities
}

\author{
G. Manfredi \\ UKAEA Government Division, Fusion (UKAEA/Euratom Fusion Association), Culham, Abingdon, Oxon, \\ OX14 3DB, United Kingdom \\ M. Shoucri \\ Centre Canadien de Fusion Magnétique, Varennes, Québec J3X 1S1, Canada \\ R. O. Dendy \\ UKAEA Government Division, Fusion (UKAEA/Euratom Fusion Association), Culham, Abingdon, Oxon, \\ OX14 3DB, United Kingdom
}

\author{
A. Ghizzo and P. Bertrand \\ LPMI, Université de Nancy-I, 54506, Nancy, France
}

(Received 28 June 1995; accepted 13 September 1995)

\begin{abstract}
An Eulerian code that solves the gyrokinetic Vlasov equation in slab geometry is presented. It takes into account the $E \times B$ and polarization drifts in the plane perpendicular to the magnetic field, and kinetic effects in the parallel direction. The finite Larmor radius is modelled by a convolution operator. The relation is established between this model and others proposed previously, and they are shown to be equivalent in the limit of long wavelengths and small Larmor radii. The code is applied to investigate ion-temperature-gradient modes in the quasi-neutral regime, with adiabatic electrons. Numerical results are reported for a wide range of parameters, including density and temperature profiles, magnetic field strength, and ion to electron temperature ratio. Normally the plasma evolves towards long wavelength structures, although in some cases (when Landau damping is very weak) more strongly turbulent regimes are observed. Test particles are used to compute diffusion coefficients both in real space and velocity space. For the most strongly turbulent regimes, particle diffusion coefficients are of order $20 \mathrm{~m}^{2} \mathrm{~s}^{-1}$. The saturation mechanism is also investigated. Many previous numerical results obtained with particle codes are confirmed, but the Vlasov Eulerian technique allows a much finer resolution of structures both in real space and velocity space. [S1070-664X(96)03001-6]
\end{abstract}

\section{INTRODUCTION}

Ion Temperature Gradient instabilities (ITG) belong to the vast class of drift instabilities. ${ }^{1}$ These involve electrostatic, low frequency waves which, under particular conditions such as the presence of a density or temperature gradient, may become unstable. Their growth rate and saturation level are both generally quite low, a circumstance that renders their numerical simulation a difficult task, for the genuine instability must be separated from the underlying numerical noise. This is particularly true for gyrokinetic particle-incell (PIC) simulations, whose high noise level has been long recognized. Fluid simulations do not suffer from this limitation, but they of course do not incorporate kinetic effects, which may play a crucial role (see however the recent attempt to include kinetic phenomena in a fluid description, by Hammett and coworkers ${ }^{2-4}$ ). Physically, ITG instabilities may play an important role in explaining the anomalous heat and particle transport observed in tokamaks. ${ }^{5-11}$ There is indeed increasing evidence from large scale gyrofluid and gyrokinetic simulations $^{12,13}$ that there is a good match between transport inferred from models of ITG-driven turbulence and measured transport in the core and confinement regions of magnetic fusion plasmas.

ITG instabilities are also called $\eta_{i}$-modes, because the crucial parameter is $\eta_{i}=d \ln T_{i} / d \ln n_{i}$. Analytic estimates ${ }^{5}$ give a critical value of $\eta_{i} \simeq 1$, above which these modes are unstable, and predict (in the case of slab geometry) that the growth rate is proportional to the cube root of $\eta_{i}$. However, these estimates are valid only within special ranges of the parameters (namely, for $\eta_{i} \gg 1$ ) and neglect important effects such as Landau damping, and of course all nonlinear phenomena.

The goal we address in this paper is a systematic numerical investigation of ITG instabilities in a shearless slab, using a gyrokinetic Vlasov code. In Vlasov (Eulerian) codes, ${ }^{14-16}$ the phase space is covered with a regular, uniform mesh, and the Vlasov equation is solved by means of a splitting algorithm. Although they consume more time and memory than the more popular PIC codes, Vlasov codes have the advantage of an extremely low level of noise. This feature allows us to obtain high resolution of coherent structures both in real space and in velocity space. Improved understanding of this aspect of plasma turbulence can thus be expected from these simulations, notwithstanding the simple slab geometry that has been used.

Some preliminary results on $\eta_{i}$-modes obtained with Vlasov codes were published in previous articles. ${ }^{15,16}$ Here, however, we make use of a slightly different model, in which the electrostatic potential is derived from the quasi-neutrality relation, instead of solving the Poisson equation. This allows us to work in the regime $\lambda_{D} \ll \rho_{i}$ (where $\rho_{i}$ is the ion Larmor radius and $\lambda_{D}$ the Debye length) which is more relevant to 


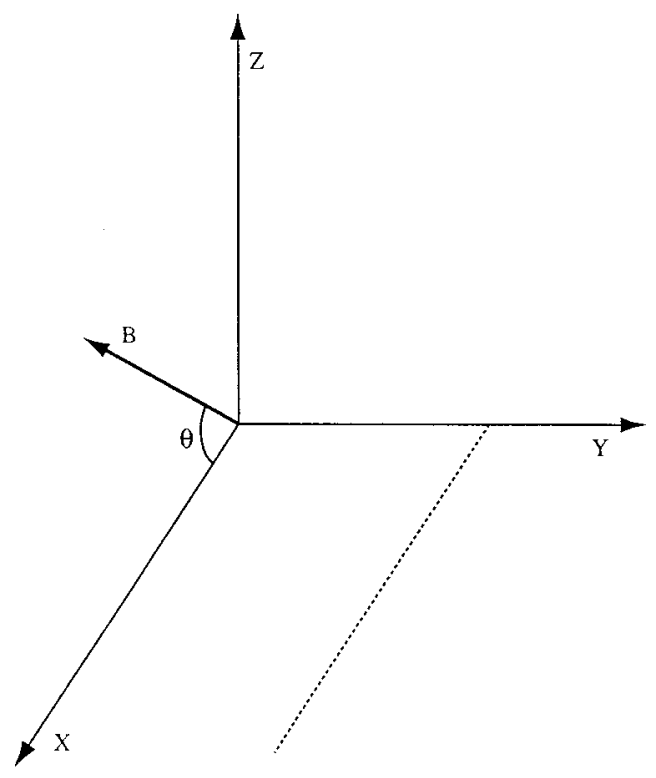

FIG. 1. Geometry of the computational box.

tokamak fusion experiments. Results of previous PIC simulations, using a model very close to the one adopted in the present paper, were published by Lee and Tang. ${ }^{17,18}$ More recently, Cohen et al. ${ }^{19}$ investigated the effect of velocity shear on ITG modes, again via PIC simulations. In our study, a velocity shear will also be present, induced by the effect of finite ion Larmor radius.

A second goal is to compare the gyrokinetic model that we have used with other models previously proposed in the literature. ${ }^{20,21}$ These models differ essentially from ours in the treatment of the polarization drift and the finite ion Larmor radius correction. However, they can be shown to be equivalent when certain, rather natural, conditions are fulfilled. It is hoped that the work presented here will contribute to the process of comparing and benchmarking numerical models of plasma turbulence in tokamaks, which is at present under way.

The paper is organized as follows. In the next section, we present the mathematical model and its relation to other, previously published, models. Conservation laws are also derived. Section III contains a brief analysis of the linear theory, as a guideline for the forthcoming simulations. In Sec. IV, we present the numerical results of the nonlinear evolution, and Sec. V deals with the computation of transport coefficients, such as the particle diffusion coefficients in real and velocity space. These results are discussed in Sec. VI, where we draw our conclusions.

\section{THE GYROKINETIC SLAB MODEL}

We consider a two-dimensional plasma slab, periodic along the $x$ "poloidal" direction, and non-periodic in the $y$ "radial" direction. The plasma is supposed to be homogeneous in the $z$ "toroidal" direction (i.e. $k_{z}=0$ ). The external magnetic field is uniform and lies in the $(x, z)$ plane, making an angle $\theta$ with the $x$ axis. The geometry of the simulation is sketched in Fig. 1. form:

The ion gyrokinetic Vlasov equation takes the following

$$
\begin{aligned}
& \frac{\partial F_{i}}{\partial t}+\nabla_{\perp} \cdot\left[\left(\vec{V}_{E}+\vec{V}_{p}\right) F_{i}\right]+V_{\|} \nabla_{\|} F_{i}+\frac{e}{m_{i}} E_{\|}^{*} \frac{\partial F_{i}}{\partial V_{\|}}=0 \\
& \vec{V}_{E}=\frac{\vec{E}^{*} \times \vec{B}}{B^{2}} \\
& \vec{V}_{p}=\frac{1}{\Omega_{i} B} \frac{d \vec{E}_{\perp}^{*}}{d t} \equiv \frac{1}{\Omega_{i} B}\left[\frac{\partial \vec{E}_{\perp}^{*}}{\partial t}+\left(\vec{V}_{E}+\vec{V}_{p}+\vec{V}_{\|}\right) \cdot \nabla \vec{E}_{\perp}^{*}\right]
\end{aligned}
$$

where $\Omega_{i}=e B / m_{i}, F_{i}=F_{i}\left(x, y, V_{\|}, t\right)$ and $\vec{E}=-\nabla \varphi$. Note that we have explicitly introduced the polarization drift in our model. We also remark that (1c) is an implicit definition of the polarization drift, since $V_{p}$ appears on the RHS of this relation: this fact will prove to be useful in the derivation of the energy integral. Appendix A gives further details of the numerical code used to solve Eq. (1a).

The asterisk appearing in Eq. (1) is a short-hand for an integral operator that takes into account the finite Larmor radius correction. Following Knorr et al. ${ }^{22,23}$ we have, for a function $h(\vec{r}), \vec{r} \equiv(x, y)$ :

$$
h^{*}(\vec{r}) \equiv \iint G\left(\vec{r}-\vec{r}_{1}\right) h\left(\vec{r}_{1}\right) d \vec{r}_{1} \equiv G * h .
$$

Equation (2) defines a convolution operator with kernel $G(\vec{r})$ and is more easily represented in wavenumber space:

$$
h^{*}(\vec{k})=G(\vec{k}) h(\vec{k}) ; \quad G(\vec{k})=\exp \left(-\frac{k_{\perp}^{2} \rho_{i}^{2}}{2}\right),
$$

where $\rho_{i}=V_{t h i} / \Omega_{i}$ is the ion Larmor radius, and, in our geometry, $k_{\perp}^{2}=k_{x}^{2} \sin ^{2} \theta+k_{y}^{2}$ (see Fig. 1). This, rather general, expression for the Larmor radius correction is derived by gyrophase averaging, and further assuming a Maxwellian distribution for the perpendicular velocity. ${ }^{22}$

In order to close the system of equations (1), we need a further relation, given by the quasi-neutrality constraint

$$
n_{i}^{*}=n_{e},
$$

where $n_{i}(x, y, t)=\int F_{i} d V_{\|}$and the electrons are taken to follow the adiabatic law

$$
n_{e}=n_{0} \exp \left(\frac{e \varphi}{T_{e}}\right) \simeq n_{0}\left(1+\frac{e \varphi}{T_{e}}\right) .
$$

The asterisk in Eq. (4) again stands for the operation defined in Eq. (2). Thus, $n_{i}^{*}$ is the particle density, while $n_{i}$ is the guiding center density. It can readily be shown that the system of equations (1), (4) and (5), with the prescription (3), and appropriate boundary conditions, possesses the following energy invariant:

$$
\begin{aligned}
W= & \frac{m_{i}}{2} \iiint F_{i} V_{\|}^{2} d V_{\|} d \vec{r}+\frac{e}{2} \iint n_{i}^{*} \varphi d \vec{r} \\
& +\frac{m_{i}}{2 B^{2}} \iint n_{i}\left|\nabla_{\perp} \varphi^{*}\right|^{2} d \vec{r} .
\end{aligned}
$$


Details of the calculation of this invariant are given in Appendix B. The first and second term in Eq. (6) represent respectively the parallel kinetic energy and the electrostatic energy. The third term can be written as $\left(m_{i} / 2\right) \iint V_{E}^{2} d \vec{r}$ and corresponds to the kinetic energy associated with drift motion.

Slightly different gyrokinetic models have been proposed elsewhere in the literature. ${ }^{20,21}$ It is therefore instructive to consider the relation of these models to the one used in this paper. The main difference lies in the treatment of the polarization drift, which is explicitly introduced in our Vlasov equation [Eq. (1a)], while other models take it into account in a modified form of the Poisson equation. Let us first rewrite Eq. (1) in the following way:

$$
\frac{d}{d t} \log F_{i}-\frac{1}{\Omega_{i} B} \frac{d}{d t} \nabla_{\perp}^{2} \varphi^{*}+\frac{e}{m_{i}} E_{\|}^{*} \frac{\partial \log F_{i}}{\partial V_{\|}}=0,
$$

where

$$
\frac{d}{d t} \equiv \frac{\partial}{\partial t}+\left(\vec{V}_{E}+\vec{V}_{p}\right) \cdot \nabla_{\perp}+V_{\|} \boldsymbol{\nabla}_{\|} \cdot
$$

We note, before continuing, that both in the original Eq. (1) and in the derivation of Eq. (7), we have used an approximation, which we now justify. Strictly, the integral operator $G$, defined in Eq. (2), does not commute with the advective derivative $d / d t$, because of the presence of terms like $\left(\vec{V}_{E}+\vec{V}_{p}\right) \cdot \nabla_{\perp}$. As illustrated in Ref. 20, Eq. (1) is obtained by assuming the distribution function to be Maxwellian in the perpendicular velocity, and then averaging all terms in $V_{\perp}$ space with a weight $J_{0}\left(k_{\perp} V_{\perp} / \Omega_{i}\right), J_{0}$ being a Bessel function. This procedure works rigorously for all terms, except those arising from the nonlinear part of the polarization drift: $\vec{V}_{E} \cdot \nabla \vec{E}_{\perp}$. For this term we have, assuming, for simplicity, that the magnetic field is parallel to the $z$ axis:

$$
\vec{V}_{E} \cdot \vec{E}_{\perp}=-\hat{z} \times \nabla \frac{E_{\perp}^{2}}{2} .
$$

Then averaging over $V_{\perp}$,

$$
\begin{aligned}
-\hat{z} \times & \int J_{0}\left(\frac{k_{\perp} V_{\perp}}{\Omega_{i}}\right) \nabla \frac{E_{\perp}^{2}}{2} e^{-V_{\perp}^{2} / 2 T} d \frac{V_{\perp}^{2}}{2} \\
& =-\hat{z} \times\left(G * \nabla \frac{E_{\perp}^{2}}{2}\right)=-\hat{z} \times \nabla\left(G * \frac{E_{\perp}^{2}}{2}\right) \\
& =-\hat{z} \times \vec{E}_{\perp}^{* *} \cdot \nabla \vec{E}_{\perp}^{* *} .
\end{aligned}
$$

In the last equality, we have defined:

$$
E_{\perp}^{* * 2} \equiv G * E_{\perp}^{2} .
$$

Thus our Eq. (1c) is rigorous only if in the nonlinear term we replace $E_{\perp}^{*}$ by $E_{\perp}^{* *}$. However, it is easy to show that, to leading order in $\rho_{i}$,

$$
E_{\perp}^{* 2}-E_{\perp}^{* * 2}=\rho_{i}^{2}\left[\frac{1}{2} \nabla^{2} E_{\perp}^{2}+\vec{E}_{\perp} \cdot \nabla^{2} \vec{E}_{\perp}\right],
$$

which shows that, for long wavelength $\left(k_{\perp} \rho_{i} \ll 1\right)$, the correction is of order $k_{\perp}^{2} \rho_{i}^{2}$. Now, since further analysis will show that this term is already of higher order in $\rho_{s} / L_{n} \ll 1$ $\left(L_{n}^{-1} \equiv \nabla n / n\right)$, we can safely neglect this correction if we are interested only in lower order terms.

Returning to Eq. (7), we perform the following transformation on the distribution function:

$$
F_{i}=\tilde{F}_{i} \exp \left(\frac{\nabla_{\perp}^{2} \varphi^{*}}{\Omega_{i} B}\right)
$$

yielding

$$
\frac{\partial \tilde{F}_{i}}{\partial t}+\left(\vec{V}_{E}+\vec{V}_{p}\right) \cdot \nabla_{\perp} \tilde{F}_{i}+V_{\|} \boldsymbol{\nabla}_{\|} \tilde{F}_{i}+\frac{e}{m_{i}} E_{\|}^{*} \frac{\partial \tilde{F}_{i}}{\partial V_{\|}}=0 .
$$

The quasi-neutrality equation becomes

$$
\begin{aligned}
n_{0} \exp \left(\frac{e \varphi}{T_{e}}\right) & =G * \exp \left(\frac{\nabla_{\perp}^{2} \varphi}{\Omega_{i} B}\right) \int \tilde{F}_{i} d V_{\|} \\
& \equiv G * \exp \left(\frac{\nabla_{\perp}^{2} \varphi}{\Omega_{i} B}\right) \tilde{n}_{i} .
\end{aligned}
$$

Expanding the exponentials in Eq. (13) and neglecting terms of order higher than $k^{2} \rho_{i}^{2}$, we obtain finally

$$
\frac{n_{i} \nabla_{\perp}^{2} \varphi}{\Omega_{i} B}-\frac{e \varphi n_{0}}{T_{e}}=n_{0}-\left(1+\frac{\rho_{i}^{2}}{2} \nabla_{\perp}^{2}\right) n_{i} .
$$

It is also usual to replace the ion density on the left hand side of Eq. (14) with its mean value $n_{0}$. Now, if we take for instance Eq. (41) in Ref. 20, and expand the modified Bessel function $\Gamma_{0}(s)$

$$
\Gamma_{0}(s) \equiv I_{0}(s) e^{-s} \simeq 1-s,
$$

where $s=k^{2} \rho_{i}^{2}$, we recover our Eq. (14) to the relevant order.

To perform dimensional analysis, we need to introduce the drift-wave ordering as follows:

$$
\begin{aligned}
& \frac{e \phi}{T_{e}} \sim \frac{\rho_{s}}{L_{n}}=\epsilon \ll 1, \quad \frac{\omega_{i}^{*}}{\Omega_{i}}=k_{\perp} \rho_{s} \frac{\rho_{s}}{L_{n}} \sim \epsilon^{2}, \\
& \rho_{s} k_{\perp} \sim \epsilon, \quad \rho_{s} k_{\|}=\cos \theta k_{x} \rho_{s} \sim \epsilon^{2}, \quad \text { for } \theta \approx \pi / 2 .
\end{aligned}
$$

Here, $\omega_{i}^{*}$ is the usual ion diamagnetic frequency, and $\rho_{s}=C_{s} \Omega_{i}^{-1}$, where $C_{s}$ is the sound speed $\sqrt{T_{e} / m_{i}}$. Equations (16) imply that the plasma natural scale is comparable to $L_{n}=(\nabla n / n)^{-1}$, and much larger than the ion Larmor radius. This can be true if the typical length in the $x$ direction satisfies $L_{x} \geqslant L_{n}$, and if an inverse cascade takes place towards long wavelength structures. Numerical simulations will confirm this point, although during the initial transient of the evolution, structures with a wavelength of order $\rho_{s}$ may appear.

By making use of Eqs. (16), it can be shown that terms in Eq. (1) scale as follows:

$$
\begin{aligned}
& \frac{\partial F_{i}}{\partial t} \sim \epsilon^{2}, \vec{V}_{E} \cdot \nabla_{\perp} F_{i} \sim \epsilon^{3}+\bar{\epsilon}^{5}, \\
& E_{\|} \frac{\partial F_{i}}{\partial v_{\|}} \sim \epsilon^{3}+\bar{\epsilon}^{5}, V_{\|} \nabla_{\|} F_{i} \sim \epsilon^{2}, \\
& \nabla_{\perp} \cdot\left(\vec{V}_{p} F_{i}\right) \sim\left(\epsilon^{5}+\bar{\epsilon}^{7}\right)+\left(\epsilon^{6}+\bar{\epsilon}^{8}\right)+\left(\epsilon^{4}+\bar{\epsilon}^{6}\right) .
\end{aligned}
$$

Manfredi et al. 
In the polarization term, the first parenthesis refers to the linear part of $\vec{V}_{p}$ (proportional to $\partial E_{\perp} / \partial t$ ), the second parenthesis refers to the term $\vec{V}_{E} \cdot \nabla \vec{E}_{\perp}$, and the third one to the term $\vec{V}_{\|} \cdot \nabla \vec{E}_{\perp}$. The implicit term $\vec{V}_{p} \cdot \nabla \vec{E}_{\perp}$ has been neglected (note however that this term is mandatory to preserve energy conservation, as shown in Appendix B). The bar over some of the terms is just short-hand to indicate that these terms come from the finite Larmor radius correction, which is of order $k_{\perp}^{2} \rho_{i}^{2}$. Note also that we have so far considered a case in which $T_{i}=T_{e}$, and thus $\rho_{s}=\rho_{i}$ : assuming a different scaling for the temperature ratio would result in additional orderings. From Eqs. (17), we see that the nonlinear polarization drift $\vec{V}_{E} \cdot \nabla \vec{E}_{\perp}$ is of higher order, and therefore its Larmor radius correction is negligible, as we had anticipated in our previous discussion.

It is interesting to note that the Vlasov equation as written in Eq. (12) is in a characteristic, non-conservative form, and therefore more suitable to be implemented in a PIC code. On the other hand, the form of Eq. (1) is conservative, and suits best the philosophy of Vlasov Eulerian codes. This is probably one reason why two different models have been chosen for different numerical implementations. However, we have seen that under quite natural assumptions, the two models must coincide.

\section{LINEAR ANALYSIS}

In this section we sketch some results obtained from linear theory. Since we shall use a simplified model, and drop several terms, we do not expect these results to be directly comparable with simulation results. However, they will provide a useful guideline in designing our computer experiments. Let us consider the Vlasov equation (1), in which we neglect finite Larmor radius and polarization drift effects, coupled to the quasi-neutrality condition, Eq. (4). We expand all quantities in Fourier series:

$$
\begin{aligned}
& F_{i}\left(x, y, V_{\|}, t\right)=F_{0}\left(y, V_{\|}\right)+\sum_{k} F_{k}\left(y, V_{\|}\right) e^{-i(\omega t-k x)}, \\
& \varphi(x, y, t)=\varphi_{0}(y)+\sum_{k} \varphi_{k}(y) e^{-i(\omega t-k x)},
\end{aligned}
$$

where $k$ stands for $k_{x}=\left(2 \pi / L_{x}\right) n$, and $n$ is an integer. We now introduce the normalization that will be used in this section and in all the subsequent numerical simulations. Time is normalized to the inverse of $\Omega_{i}=e B / m_{i}$, velocity is normalized to $C_{s}=\sqrt{T_{e} / m_{i}}$, space is normalized to $\rho_{s}=C_{s} / \Omega_{i}$, and the potential is normalized to $T_{e} / e$. All numerical values are understood to be expressed in these units. With this notation the equilibrium quantities appearing in Eq. (18) are

$$
\begin{aligned}
& F_{0}\left(y, V_{\|}\right)=\frac{n_{0}(y)}{\sqrt{2 \pi T(y)}} \exp \left(-\frac{V_{\|}^{2}}{2 T(y)}\right), \\
& \varphi_{0}(y)=0 .
\end{aligned}
$$

Here $n_{0}(y)$ and $T(y)$ are the density and initial temperature profiles. The equilibrium potential is zero since, at $t=0$, we have $n_{i}=n_{e}=n_{0}$ : a non-zero equilibrium potential could be present, had we considered a finite ion Larmor radius.

With the help of Eqs (18) we arrive at the desired dispersion relation

$$
n_{0}(y)+\int \frac{\partial F_{0} / \partial V_{\|}}{\omega / k_{\|}-V_{\|}} d V_{\|}-\tan \theta \int \frac{\partial F_{0} / \partial y}{\omega / k_{\|}-V_{\|}} d V_{\|}=0,
$$

where $k_{\|}=k \cos \theta$. In order to investigate the long wavelength behaviour, we expand the denominator $\left(\omega / k_{\|}-V_{\|}\right)^{-1}$ and carry out the integrals. Resonance effects at $V_{\|}=\omega / k_{\|}$, which give rise to Landau damping, are neglected here, although not of course in our full numerical treatment. Finally we arrive at the dimensionless expression:

$$
3 T s^{4}-\frac{\eta_{i}-2}{2} \frac{\tan \theta}{L_{n}} T s^{3}+s^{2}+\frac{\tan \theta}{L_{n}} s-1=0,
$$

where $s=k_{\|} / \omega \ll 1, \eta_{i}=d \log T / d \log n_{\mathrm{o}}$. From Eq. (21) we see that $\alpha \equiv \tan \theta / L_{n}$ is a crucial parameter. Several limiting cases can be analyzed from Eq. (21). For very small $s$

$$
\omega=\omega_{i}^{*} \equiv\left(k_{\perp} \rho_{s}^{2} / L_{n}\right) \Omega_{i},
$$

where $k_{\perp}=k \sin \theta$, and we have restored dimensional units. When $\alpha \ll 1$, we recover sound waves $\omega=C_{s} k_{\|}$. It is also clear that, in order to obtain unstable solutions, a temperature gradient (i.e. a non-zero $\eta_{i}$ ) must be present.

The case $\eta_{i} \gg 1$ is particularly interesting. Keeping $\alpha$ and $T$ fixed, we can neglect the term in $s^{4}$. The result is a third degree algebraic equation that admits an unstable solution with:

$$
R e s \sim I m s \sim\left(\frac{1}{\alpha \eta_{i} T}\right)^{1 / 3}
$$

or, in terms of the dimensional frequency

$$
\begin{aligned}
& \operatorname{Im}\left(\frac{\omega}{\Omega_{i}}\right)=\frac{2 \sqrt{3}}{3}\left(\frac{\alpha}{2} \eta_{i} \rho_{s} \frac{T_{i}}{T_{e}}\right)^{1 / 3} k_{\|} \rho_{s}, \\
& \operatorname{Re}\left(\frac{\omega}{\Omega_{i}}\right)=2\left(\frac{\alpha}{2} \rho_{s} \eta_{i} \frac{T_{i}}{T_{e}}\right)^{1 / 3} k_{\|} \rho_{s} .
\end{aligned}
$$

A little algebra shows that the left hand side of Eq (22) yields:

$$
\omega \sim\left(\omega_{i}^{*} C_{s}^{2} k_{\|}^{2} \eta_{i}\right)^{1 / 3}
$$

as obtained, for example in Ref. 5.

Another interesting case is the flat density profile, for which $L_{n} \rightarrow \infty, \eta_{i} \rightarrow \infty, \alpha \rightarrow 0$, but $L_{T}$ is finite. Equation (21) reduces to

$$
\frac{T^{\prime} \tan \theta}{2} s^{3}-s^{2}+1=0
$$

$\left(T^{\prime}=d T / d y\right)$, which possesses an unstable root when $T^{\prime} \tan \theta>4 \sqrt{3} / 9$. This means that for $\theta$ approaching $90^{\circ}$, an unstable solution always exists, even for a very small temperature gradient. The growth rate, in the regime where $T^{\prime} \tan \theta \gg 1$, is given by

$$
\operatorname{Im} \omega=\frac{2 \sqrt{3}}{3}\left(\frac{T^{\prime} \tan \theta}{2}\right)^{1 / 3} k_{\|} .
$$


Restoring dimensional quantities, Eq. (25) can be expressed in a form similar to that of Eq. (23):

$$
\omega \sim\left(\omega_{* T_{i}} C_{s}^{2} k_{\|}^{2} \eta_{i}\right)^{1 / 3}
$$

where $\omega_{* T_{i}}=k_{\perp} \rho_{i}^{2} \Omega_{i} / L_{T}$.

\section{NUMERICAL RESULTS}

In this section we perform extensive numerical simulations of the ITG modes, by varying the crucial physical parameters involved in the governing equations. The model used throughout this section is the one described by Eqs. (1) to (5) in Sec. II, and the density and temperature profiles adopted throughout this paper are:

$$
\begin{aligned}
& n_{0}(y)=N_{0}\left\{1-\frac{\Delta_{n}}{2}\left[1-\tanh \left(\beta_{n} y\right)\right]\right\}, \\
& T(y)=T_{i 0}\left\{1-\frac{\Delta_{T}}{2}\left[1-\tanh \left(\beta_{T} y\right)\right]\right\} .
\end{aligned}
$$

The density profile is fixed for the electrons and also constitutes the initial (guiding-center) density for the ions. The temperature profile intervenes in the ion initial condition as illustrated by Eq. (19). Note that these profiles do not give a constant $L_{n}^{-1}$ or $L_{T}^{-1}$, but have a bell-like shape as shown in Fig. 2. The maximum is reached at $y=0$, where $L_{n}^{-1}=\Delta_{n} \beta_{n} /\left(2-\Delta_{n}\right)$. This fact will render the comparison with analytical results more cumbersome, but has the advantage of being more easily implemented numerically, since the

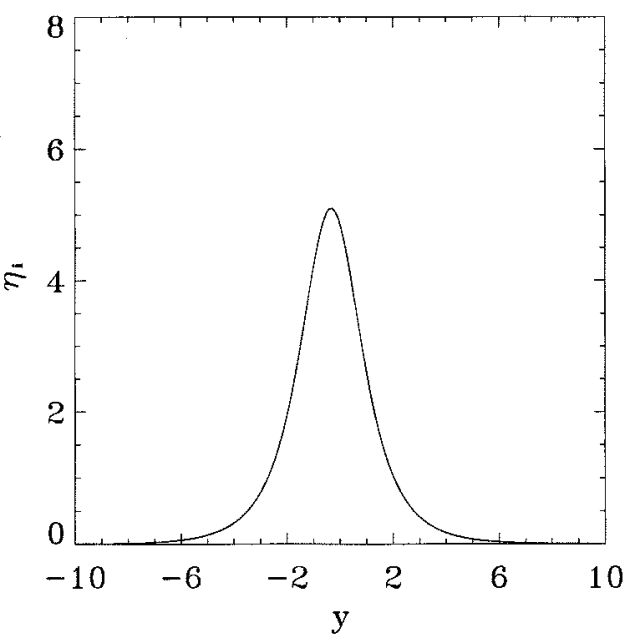

FIG. 2. Typical profile of $\eta_{i}(y)=d \log T_{i} / d \log n_{i}$.

profiles become flat at $y= \pm \infty$, and the plasma can be supposed to be homogeneous outside the computational box. The instability is started up by adding a small perturbation (with an amplitude $\epsilon \simeq 10^{-3}$ ) to the first three modes of the ion density in the $x$ direction.

In the first group of simulations, we consider both a density and temperature gradient, thus yielding a finite $\eta_{i}$. The physical parameters, expressed as usual in dimensionless form, are:
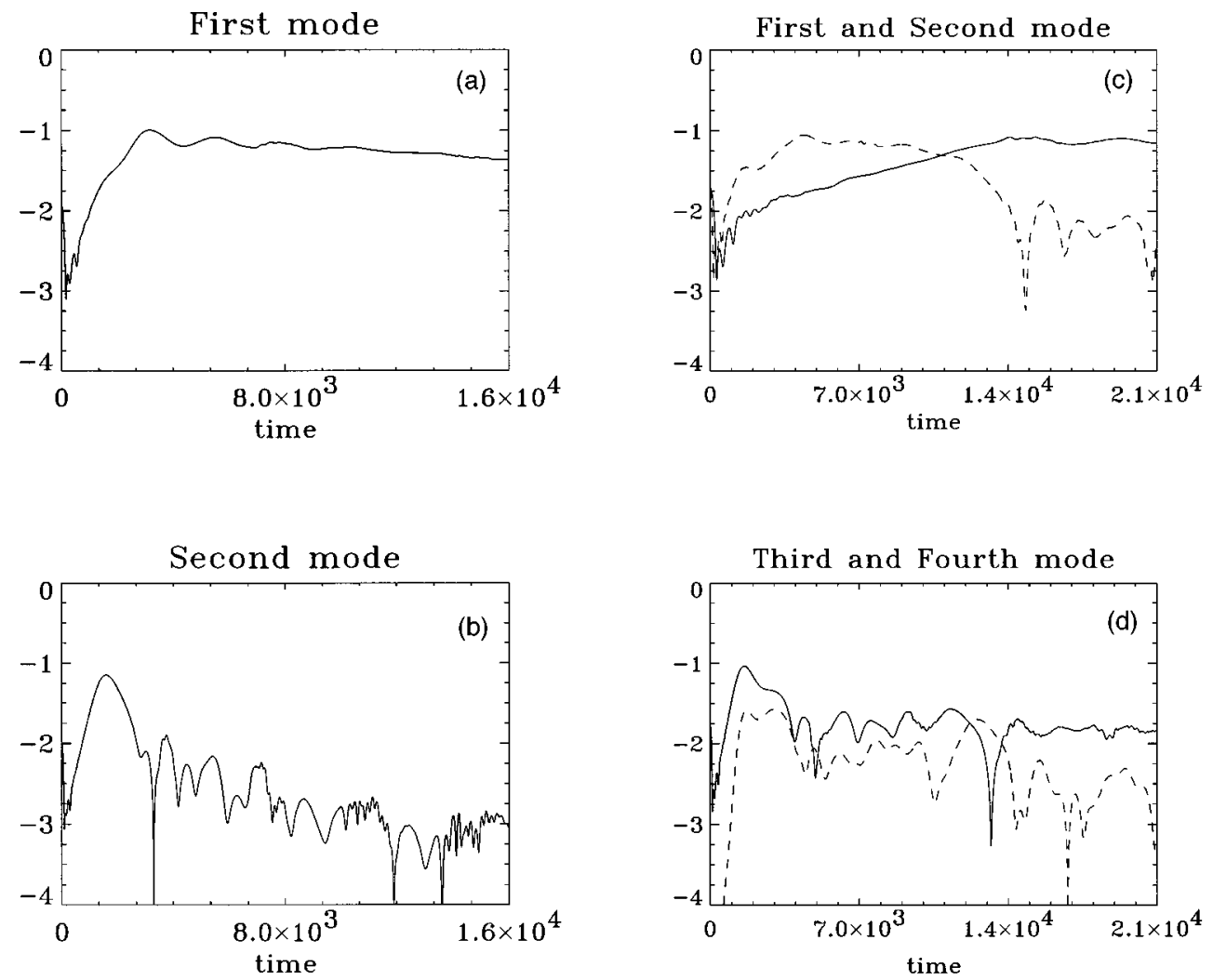

FIG. 3. Time evolution of the modes of the electrostatic potential for $\theta=88^{\circ}, L_{x}=25$ and mode $k_{0}=2 \pi / 25$ (a); $L_{x}=25$, mode $2 k_{0}$ (b); $L_{x}=50$, modes $k_{0}=2 \pi / 50$ (solid line) and $2 k_{0}$ (broken line) (c); $L_{x}=50$, modes $3 k_{0}$ (solid line) and $4 k_{0}$ (broken line) (d). Here, and in the following, the scale is logarithmic in base ten. 

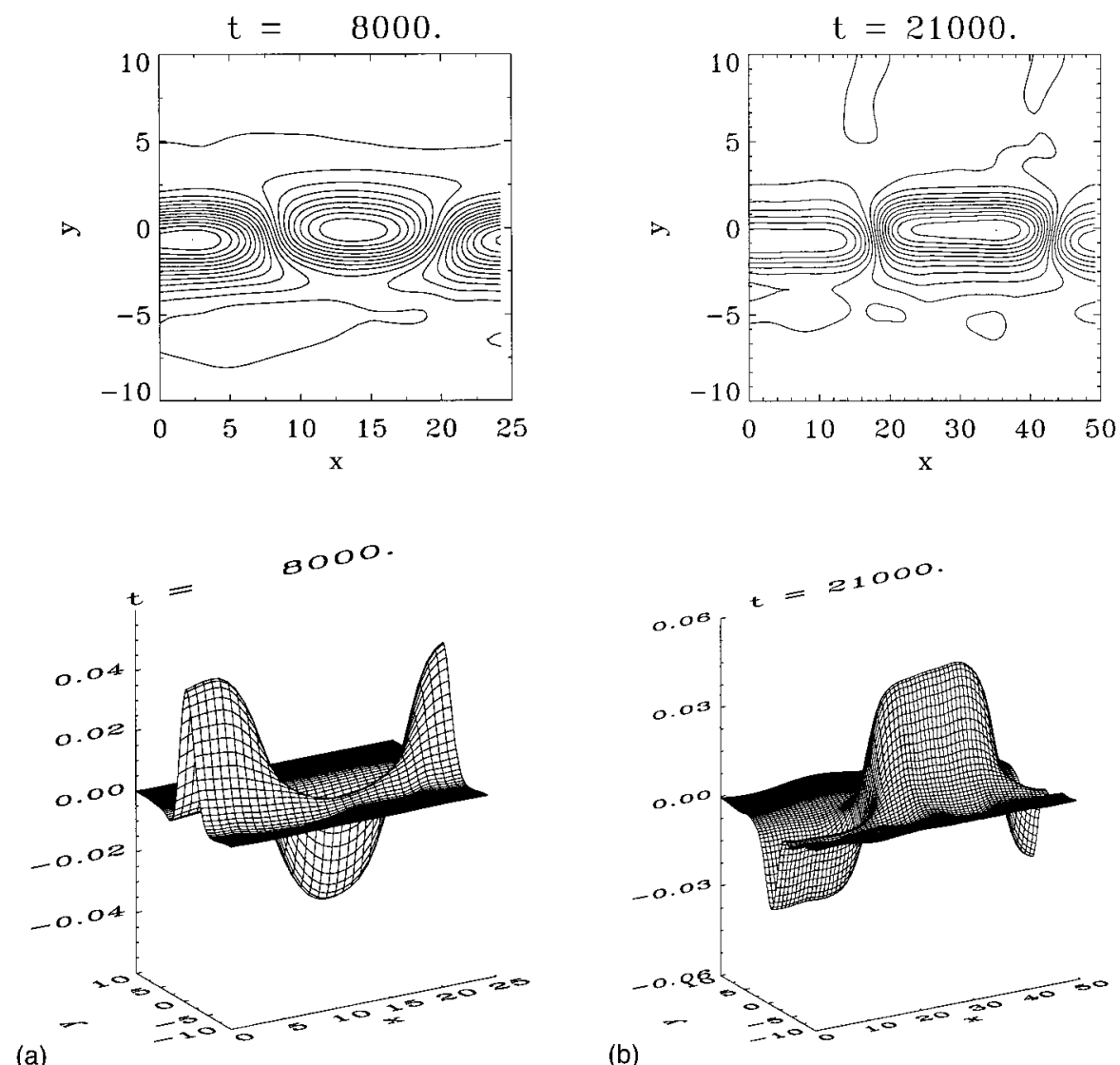

FIG. 4. Contour plot and three-dimensional view of the potential at the end of the simulation for $\theta=88^{\circ}$ and $L_{x}=25$ (a) and $L_{x}=50$ (b).

$$
\begin{array}{ll}
T_{i 0}=T_{e} ; & \theta=88^{\circ} ; \quad \Delta_{n}=0.3 ; \quad \beta_{n}=0.4 ; \\
\Delta_{T}=0.7 ; \quad \beta_{T}=0.8
\end{array}
$$

giving a maximum value of $\eta_{i}=6.1\left(L_{n} \simeq 14, L_{T} \simeq 2.3\right)$. The numerical parameters must be adjusted according to the natural space and time scales of the system. Equation (22) can be used to determine the order of magnitude of a typical frequency. With the above physical parameters we obtain $\omega \sim 0.02 \Omega_{i}$ for the fundamental mode $k_{0}=2 \pi / L_{x}$ $=2 \pi / 25 \simeq 0.25$. Accordingly, we have taken a time step $\Omega_{i} \Delta t=4$. The computational box is as follows: $0<x<L_{x}$; $-L_{y}<y<L_{y} ; \quad-V_{\max }<V_{\|}<V_{\text {max }}$, with $L_{x}=25 \rho_{s}$ or $L_{x}=50 \rho_{s}, L_{y}=10 \rho_{s}, V_{\text {max }}=5 C_{s}$. The number of points in each direction is $N_{x} \times N_{y} \times N_{v}=32 \times 80 \times 100=2.56 \times 10^{5}$. This gives a resolution of $\Delta x=0.78 \rho_{s}$, $\Delta y=0.25 \rho_{s}=0.11 L_{T}, \Delta V_{\|}=0.1 C_{s}$. For this value of $\theta=88^{\circ}$, Landau damping provides an effective source of dissipation, which allows such a relatively low spatial resolution.

A very common feature, observed in many fluid and kinetic simulations of plasmas ${ }^{13,24}$ as well as in laboratory experiments $^{25}$, is the so-called inverse cascade. Small scale vortices are created in the transient regime, but they rapidly coalesce to give rise to larger vortices, and finally only large scale structures persist. In other words, the system selects the longest wavelength allowed by the imposed boundary conditions. In order to check the existence of this phenomenon, we have performed two simulations with, respectively, $L_{x}=25 \rho_{s}$ and $L_{x}=50 \rho_{s}$. In Fig. 3 we show the time evolution of several modes of the electrostatic potential $\left|\varphi_{k}(t)\right|$ integrated over the $y$ direction. For the case $L_{x}=25 \rho_{s}$, the first mode $\left(\rho_{s} k_{0}=2 \pi \rho_{s} / L_{x} \simeq 0.25\right)$ saturates around $\Omega_{i} t=3000$, while higher order modes remain at a lower level after saturation. For the case $L_{x}=50 \rho_{s}$, the second mode (with now $k_{1}=0.25$ ) initially dominates, saturating around $t=6000$, but finally, after a long plateau, it drops quite abruptly at $t=13000$. Meanwhile the first mode $\left(k_{0}=0.125\right)$ has satu-

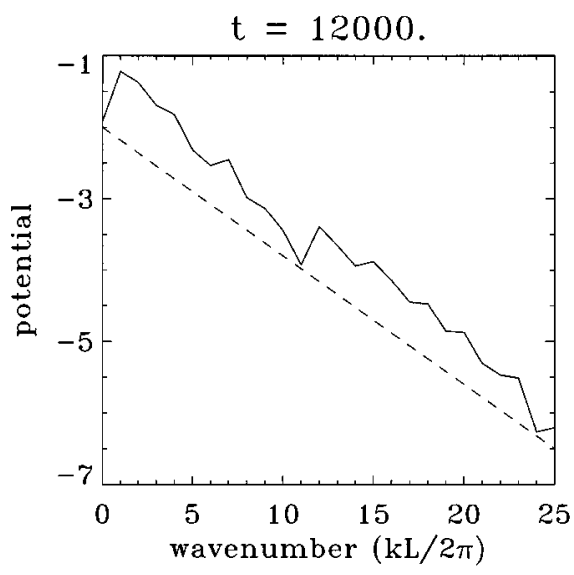

FIG. 5. Wavenumber spectrum of the electrostatic potential, integrated along the non-periodic direction, at time $t=12000$. The scale is logarithmic in base ten. The straight broken line has a slope $-0.18 \log k$. 
First mode

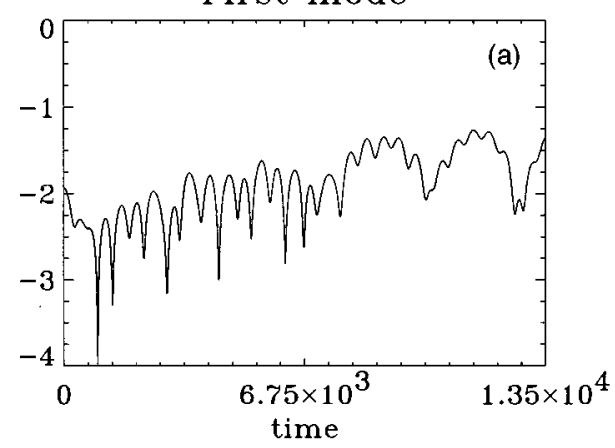

Second mode

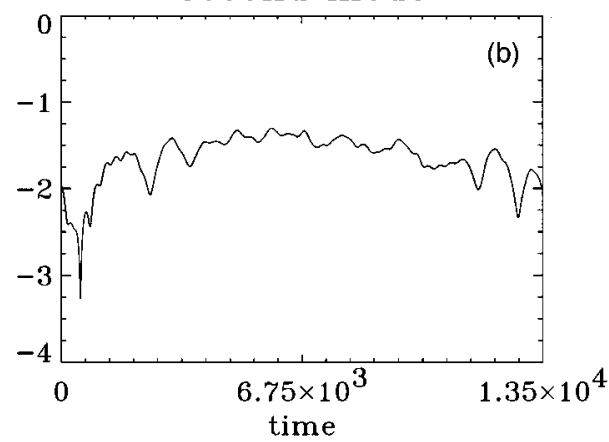

Third mode

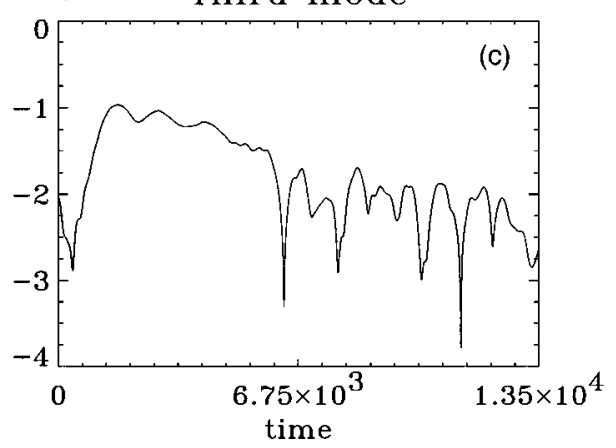

Fourth mode

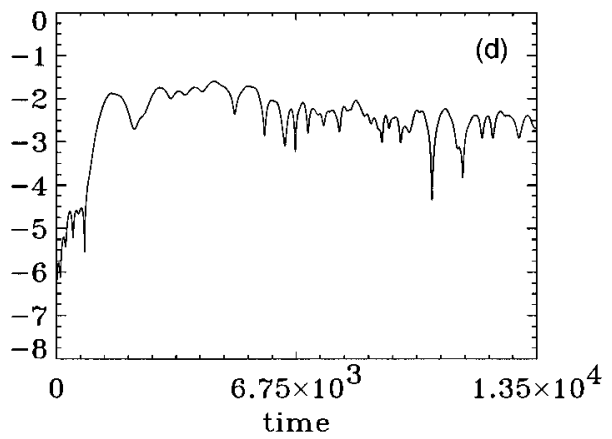

FIG. 6. Time evolution of the modes of the electrostatic potential for $\theta=89.5^{\circ}$ and $L_{x}=25$. The plots correspond to wavenumbers $k_{0}=2 \pi / 25$ (a); $2 k_{0}$ (b); $3 k_{0}$ (c) and $4 k_{0}(\mathrm{~d})$.
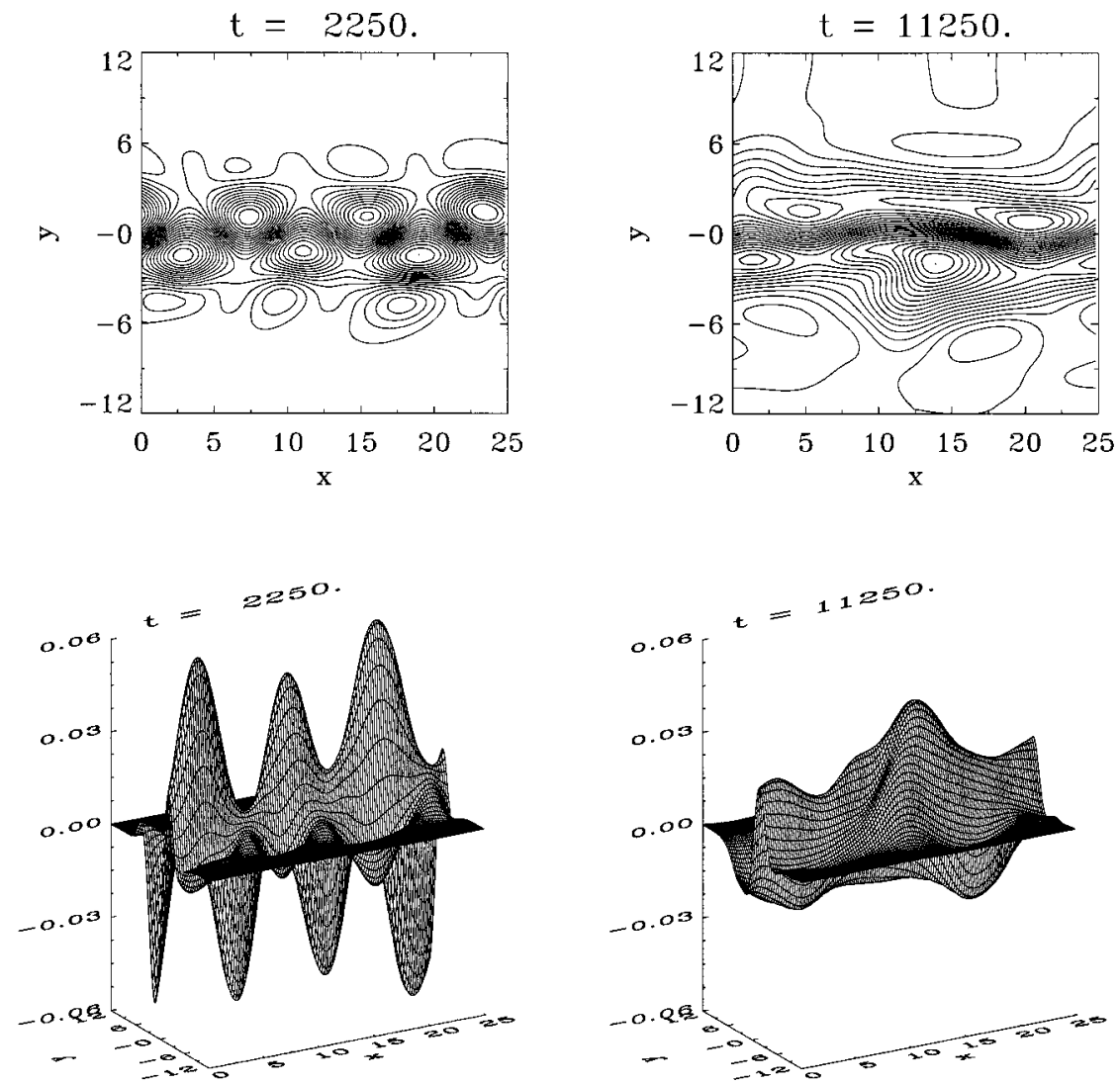

(a)

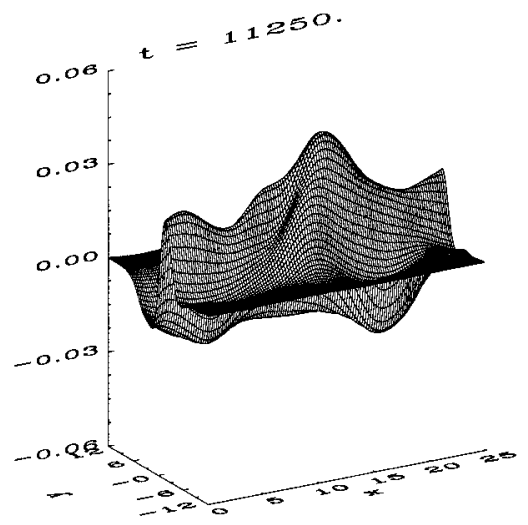

(b)

FIG. 7. Electrostatic potential for the same case as Fig. 6, shown at different times: $t=2250$ (a); $t=11250$ (b). 
rated at $t=14000$, and remains dominant in the subsequent evolution. A contour plot of the potential at the end of the simulation (Fig. 4) shows that, in both cases, a large scale structure survives. The wavenumber spectrum of the electrostatic potential integrated over $y$ (shown in Fig. 5) seems to obey an exponential law $10^{-\mu k}$ (with $\mu \simeq 0.18$ ) from the early stage of the simulation: subsequently, the spectrum is found to be stabilized and only small fluctuations persist. This rapid fall of the spectrum is quite unusual, compared to the power law behaviour of fluid turbulence, and invites future investigation. As a first step towards an explanation, we note that Landau damping affects both high and low wavenumber structures, while collisional damping is localized in the high $k$ part of the spectrum. This simple fact could prevent the existence of an inertial range of wavenumbers in which the mechanism for dissipation can effectively be ignored. Finally, we note that, in these simulations, the distribution function in velocity space (not shown here) has undergone little change. The evolution is therefore essentially linear, as far as the parallel velocity non-linearity is concerned.

As a second example, we study a case for which $\theta=89.5^{\circ}$ and $L_{x}=25$, while all other parameters are the same as in the previous simulation. With this value of the angle $\theta$, the ratio of the poloidal to the toroidal magnetic field is $B_{x} / B_{z}=k_{x} / k_{z}=\tan ^{-1} \theta=0.0087$, and the parallel wavenumber is $k_{\|} / k_{x}=\cos \theta=0.0087$. We also have $\alpha=\tan \theta / L_{n} \simeq 8.1$. The first modes of the electric potential are shown in Fig. 6. Comparing with the previous case $\theta=88^{\circ}$, we remark that the third and fourth harmonics undergo a very fast initial growth (note that only the three first modes were initially perturbed with an amplitude $\epsilon=0.001)$. However, these higher order modes quickly saturate, and, in the long run, the fundamental mode $k_{0}=2 \pi / L_{x}$ becomes dominant. This behaviour can be explained as follows. High wavenumber modes would be the most unstable ones in the absence of Landau damping, as shown by Eq. (22). However, for small enough values of $\theta$ (corresponding to larger $k_{\|}$and hence lower parallel phase velocity) Landau damping prevents these small scale structures developing, while for $\theta$ close to $90^{\circ}$ they can dominate the initial evolution. Note that an angle of $\theta=87^{\circ}$ must already be considered as small, since we have observed that virtually only the fundamental mode survives. In fact, for the parameters of the previous simulations, the parallel phase velocity computed from the numerical results is $\omega / k_{\|} V_{t h} \simeq 0.64$ when $\theta=88^{\circ}$ and $\omega / k_{\|} V_{t h} \simeq 2.5$ when $\theta=89.5^{\circ}$. For $\theta=85^{\circ}$, and the same parameters as used above, all modes are damped, and the system is stable.

Returning to the case $\theta=89.5^{\circ}$, Fig. 7 presents a contour plot and a tridimensional view of the electrostatic potential at various times. We note the formation of small wavelength structures at the initial stage of the simulation. The final state is definitely more strongly turbulent than in the case $\theta=88^{\circ}$. This is a general feature we have observed in these and other examples: as the angle $\theta$ approaches $90^{\circ}$, a strongly turbulent regime, with short wavelength vortices, begins to appear. For this reason, we often added a small dissipative term of the form $\nu \nabla_{\perp}^{2} F_{i}$ to the Vlasov equation, Eq. (1a). In the present case we have $\nu=5 \times 10^{-5} \Omega_{i} \rho_{s}^{2}$. In addition, we have in-

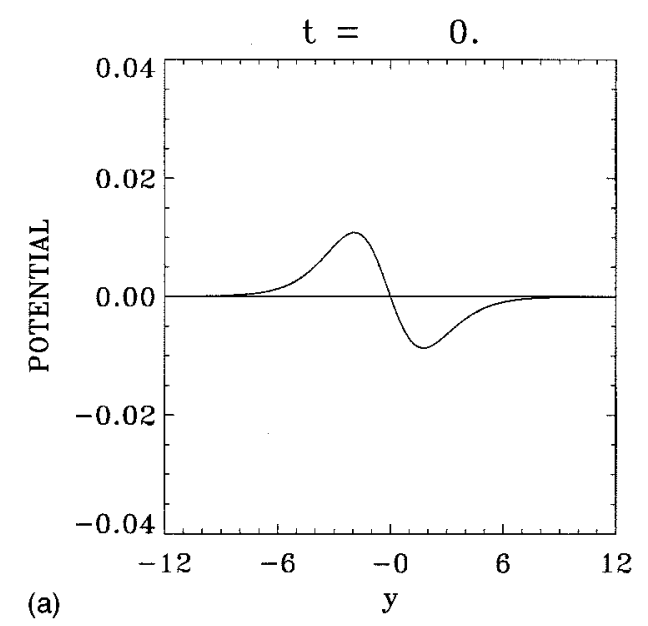

(a)
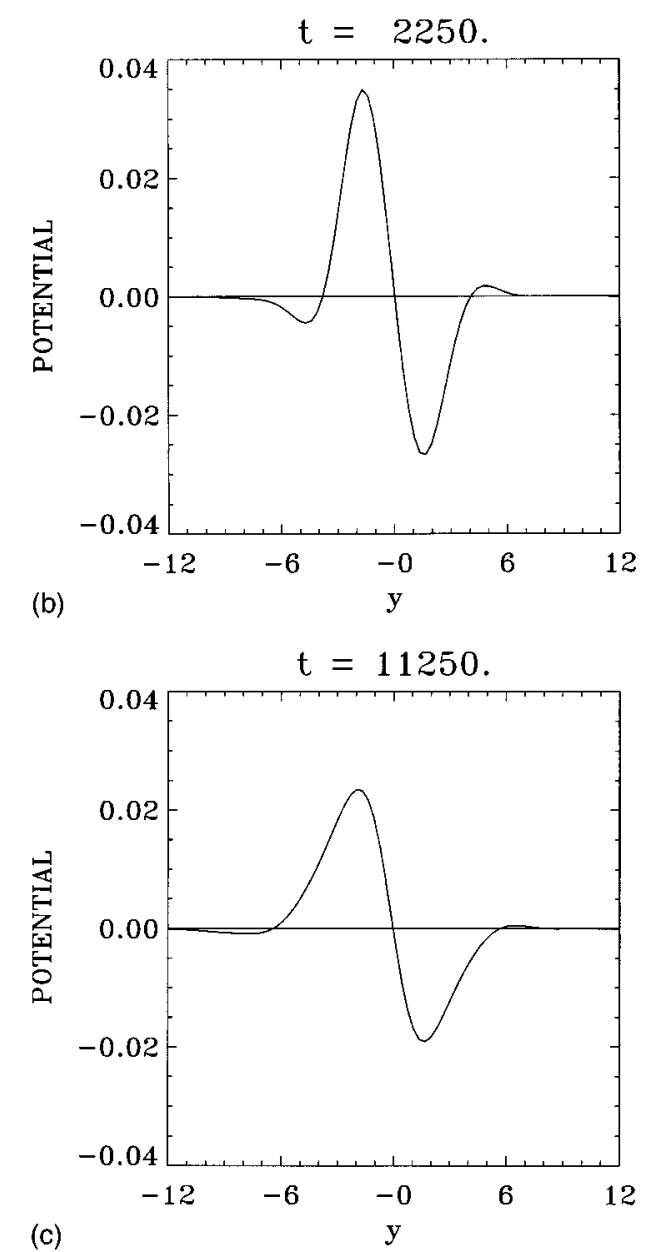

FIG. 8. Electrostatic potential, averaged along the $x$ direction, for the same case as Fig. 6: $t=0$ (a); $t=2250$ (b); $t=11250$ (c).

creased the resolution in the $x$ direction, which we found to be most affected by the small scale structures, taking $N_{x} \times N_{y} \times N_{v}=128 \times 80 \times 80$. It is important to note that the most severe constraint on $N_{v}$ does not come from accuracy, but from the recurrence time $\tau=2 \pi / k_{x} \Delta V \cos \theta$. This is the reason why for $\theta \approx 90^{\circ}$ we can take a smaller number of points in velocity space. In Fig. 8 we show the electrostatic potential, now averaged along the $x$ direction. The initial potential is non-zero because of finite Larmor radius effects 


\section{PRESSURE}

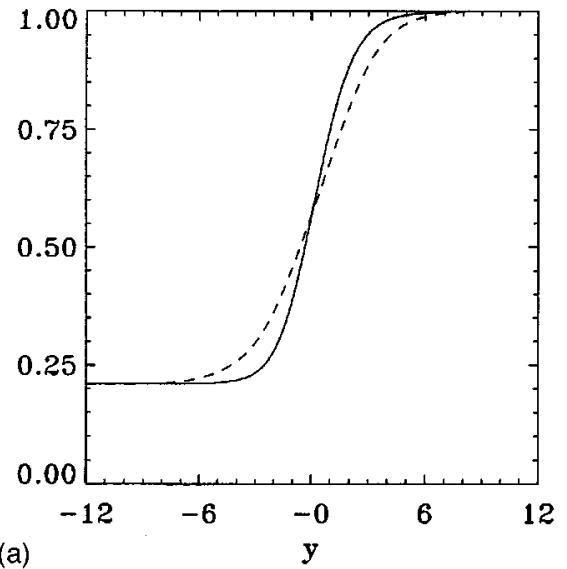

(a)

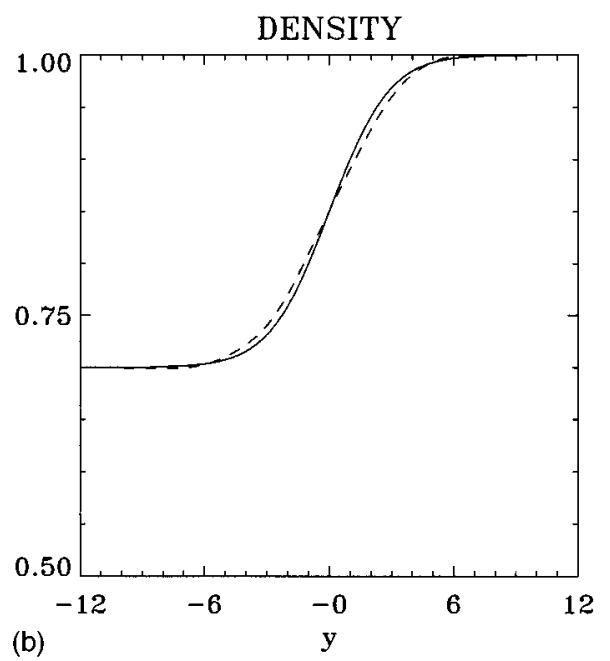

(b)

FIG. 9. Pressure profile (a) and density profile (b) as a function of $y$, at $t=0$ (solid line) and at $t=13500$ (broken line).

(remember that in our model the potential is proportional to the fluctuating part of the ion density). The potential increases in the early stage of the simulation, then it relaxes to an almost constant value (actually, there is a slight steady growth, but this is due to the small dissipation term, which progressively flattens the ion density gradient). This modification in the mean potential cannot be due to the $E \times B$ drift (which, combined with the adiabaticity law, yields no net current across the magnetic field). However, in the presence of a finite Larmor radius and polarization drift, transport in the $y$ direction is allowed, and the potential profile can evolve.

Another important question is the mechanism that provides saturation of the ITG instability. This is addressed in Fig. 9, which shows the density and the parallel pressure $p_{\|}=n T_{\|}$, averaged along $x$, as a function of $y$, at the beginning and at the end of the simulation. We see that the initial steep pressure profile becomes considerably smoother, whereas the density profile undergoes little change. The system thus evolves to a state in which a temperature gradient (although now a stable one) still exists.

In order to investigate the importance of the viscosity term, we have repeated our first simulation $\left(\theta=88^{\circ}, L_{x}=25\right)$
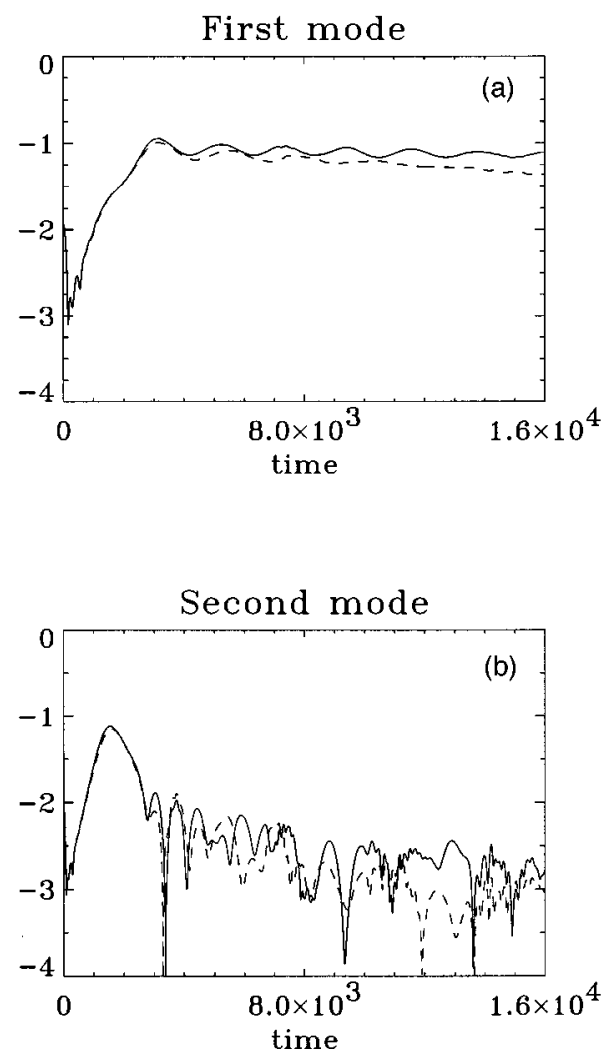

FIG. 10. Modes of the electrostatic potential for $\nu=0$ (solid line) and $\nu=5 \times 10^{-5}$ (broken line), for a simulation with $\theta=88^{\circ}, L_{x}=25, \eta_{i} \simeq 6$. The plots correspond to wavenumbers $k_{0}=2 \pi / 25$ (a) and $2 k_{0}$ (b).

for a longer time $\left(\Omega_{i} t=16000\right)$, in two cases where $\nu=0$ and $\nu=5 \times 10^{-5}$ respectively. The plot of the Fourier modes (Fig. 10) shows that they are little influenced by a small viscosity. However, things are quite different in the $y$ direction, due to the presence of a density gradient, as we anticipated earlier. The potential, averaged along $x$, is presented in Fig. 11 for both cases. When $\nu=0$, the potential stays at a low level, and takes on a "Mexican Hat" shape. When $\nu>0$, the viscosity flattens the density profile, which departs more strongly from the equilibrium profile $n_{0}$, resulting in an increase of the potential. The shape of the potential profile remains close to the initial one, although at a higher level. This simple factthat viscosity can enhance the electric field in the presence of a density gradient—could be an important issue in edge plasma physics, where all these ingredients play a crucial role.

The growth rates obtained in the previous simulations are smaller than the analytical estimate, Eq. (22), which gives Im $\omega \sim 0.0186 \Omega_{i}$ for the typical parameters of our first simulation and for the fundamental harmonic. This discrepancy is mainly due to Landau damping and finite Larmor radius effects (the latter also acts to reduce the growth rate), which are not included in the calculations leading to Eq. (22): indeed, our order-of-magnitude calculation for the parallel phase velocity, yielding $\omega / k_{\|} V_{t h} \simeq 2.5$, proves that Landau damping is not negligible even for $\theta=89.5^{\circ}$. We also note that the growth rates that we compute are not local, since they are averaged over the non-periodic direction, and 


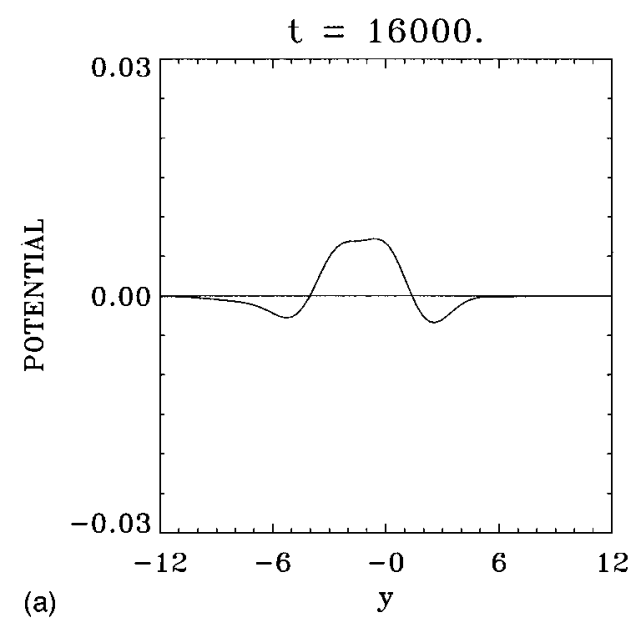

(a)

$\mathrm{t}=16000$.

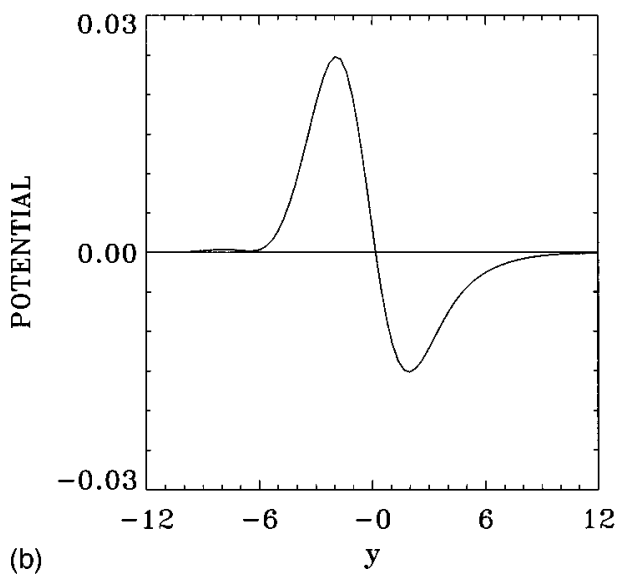

FIG. 11. Plot of the electrostatic potential at $t=16000$ averaged along the $x$ direction for the case of Fig. 10, and $\nu=0$ (a), $\nu=5 \times 10^{-5}$ (b).

that our value of $\eta_{i}$ is a function of $y$ (see Fig. 2). As a test for the accuracy of the code, we have thus performed a simulation with $\theta=90^{\circ}$ and $\rho_{i}=0.15 \rho_{s}$. In this case, the density profile is stable, and the plasma oscillates at the diamagnetic frequency $\omega_{i}^{*}=k_{x} \rho_{s}^{2} \Omega_{i} / L_{n}$. The theoretical frequency $\omega_{i}^{*}$ $=0.0178 \Omega_{i}$ is very close to the frequency observed in the simulation.

In order to investigate the effect of a finite Larmor radius on the instability, we have performed a simulation with the following dimensionless parameters:

$$
\begin{aligned}
& L_{x}=25 ; \quad \theta=88^{\circ} ; \quad \Delta_{n}=0.3 ; \quad \beta_{N}=0.4 ; \\
& \Delta_{T}=0.7 ; \quad \beta_{T}=0.8 ; \quad \rho_{i}=0.4 \rho_{s} .
\end{aligned}
$$

These are identical to the parameters of our first simulation (see Fig. 3), except for the smaller ion Larmor radius. We also added a viscosity $\nu=3 \times 10^{-4}$. The time evolution of the first four modes (Fig. 12) shows a very rapid growth of the third and fourth harmonics, resulting in a more strongly turbulent regime. Generally speaking, effects due to the finite perpendicular ion temperature seem to play an important role in stabilizing small scale structures.

We now consider the case of a flat density profile, $n_{0}=1$. In this situation, the smoothing operation that accounts for the finite ion Larmor radius [Eq. (2)] has no effect, at least
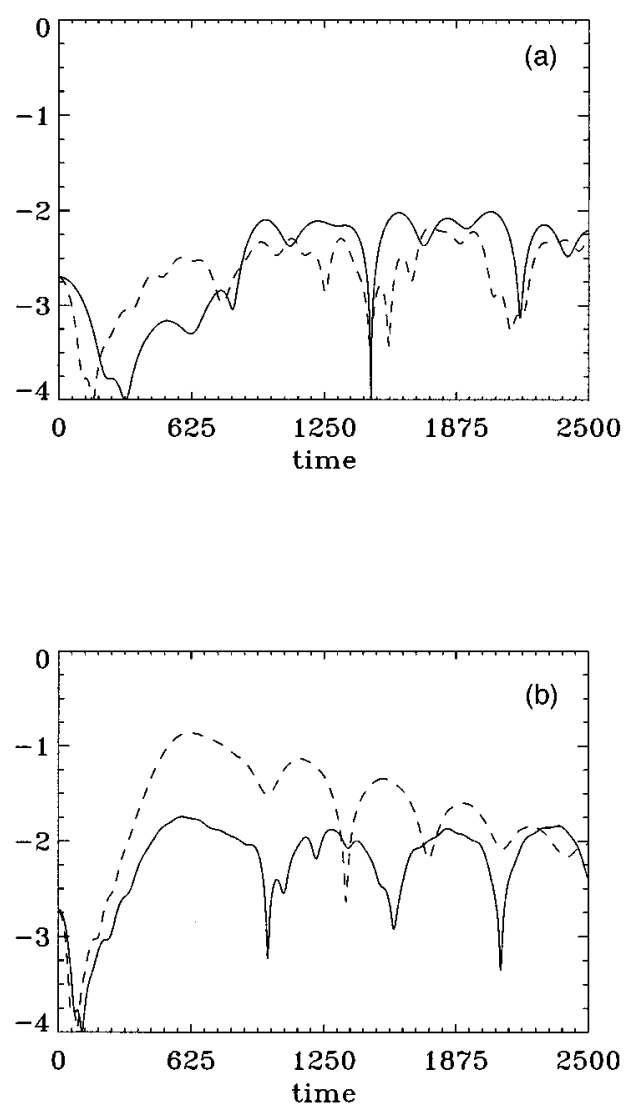

FIG. 12. Time evolution of the modes of the electrostatic potential, for the case of a smaller Larmor radius, $\rho_{i}=0.4 \rho_{s}$, for wavenumbers $k_{0}=2 \pi / 25$ (solid line) and $2 k_{0}$ (broken line) (a); $3 k_{0}$ (solid line) and $4 k_{0}$ (broken line) (b).

initially, on the ion density $n_{i}(t=0)=n_{0}=1$. Thus we can expect behaviour close to that observed when $\rho_{i} \ll \rho_{s}$, i.e. enhanced instability and growth of many short wavelength modes. We shall concentrate on a limiting case, which displays interesting features. The dimensionless physical parameters are:

$$
\begin{array}{llll}
\theta=89.9^{\circ} ; & T_{i 0}=T_{e} ; & \rho_{i}=\rho_{s} ; & \Delta_{T}=0.4 ; \\
\beta_{T}=0.4 ; & \nu=10^{-4} ; & L_{x}=25 ; & L_{T}=10 .
\end{array}
$$

Note that $\theta$ is close to $90^{\circ}$, which gives $B_{x} / B_{z} \approx k_{\|} / k_{x} \approx 0.0017$. We shall see however that an instability still exists: this is most surprising since, when $\theta$ is rigorously equal to $90^{\circ}$, any initial condition with a flat density is a stationary solution $(\omega=0)$, even when it is perturbed in the $x$ direction (this is because, for $n_{0}=1$, the initial state is only a function of $x$ through the perturbation). This feature has been accurately checked with the numerical code over many $\Omega_{i}^{-1}$. The evolution of the modes (Fig. 13) shows that short wavelength harmonics dominate the early stages of the simulation. Then, around $\Omega_{i} t=5500$, the first mode suddenly grows very quickly, and by $\Omega_{i} t=10000$ it has become the dominant one. Contour levels of the potential (Fig. 14) reveal strongly turbulent behaviour, with intricate structures that spread all over the domain, whereas, in previous cases, the instability was concentrated in the region of maximum $\eta_{i}$. In the long run, small scale structures are wiped away by the 
First mode
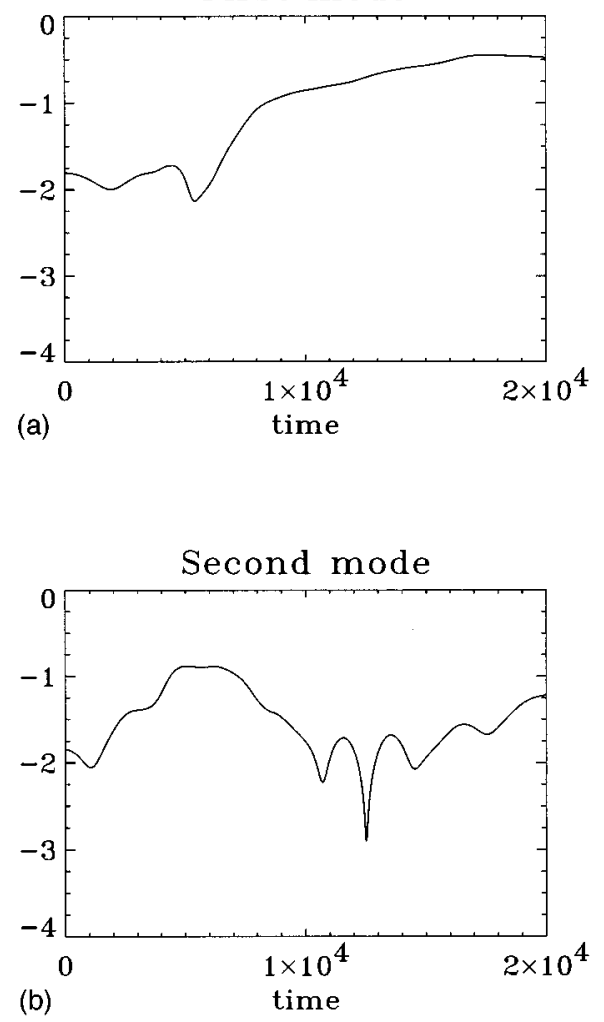

Third mode

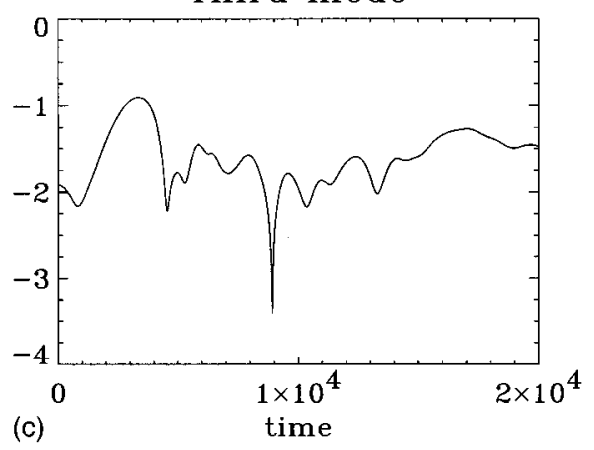

Fourth mode

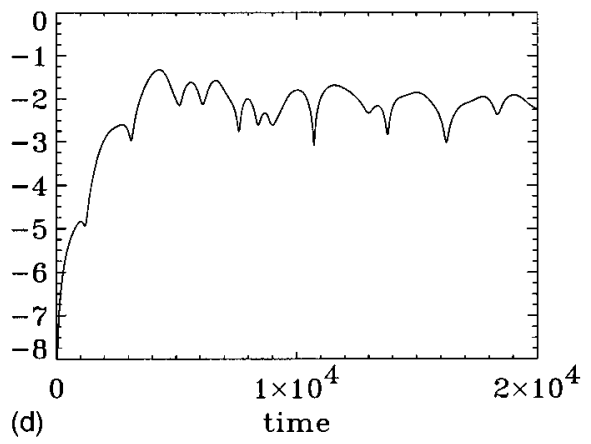

FIG. 13. Time evolution of the modes of the electrostatic potential in the case of a flat density profile and $\theta=89.9^{\circ}$, for wavenumbers $k_{0}=2 \pi / 25$ (a); $2 k_{0}(\mathrm{~b})$; $3 k_{0}(\mathrm{c})$ and $4 k_{0}(\mathrm{~d})$.

viscosity, but large scale vortices persist up to $\Omega_{i} t=20000$. Finally, the distribution function at the end of the simulation is shown in Fig. 15: a large vortex is still present at $\Omega_{i} t=20000$, which represents a non-negligible modulation of the initial Maxwellian (about 10\% of its maximum). Again, it is remarkable that such phase space structures can develop with a parallel wavenumber of about $k_{\|}=4 \times 10^{-4} \rho_{s}^{-1}$.

This simulation, and the one previously performed with $\rho_{i} \ll \rho_{s}$, support the view that ITG instabilities grow faster and saturate with a more strongly turbulent state when the equilibrium potential $e \varphi_{0} / T_{e}$ is small or zero. In order to have $\varphi_{0} \neq 0$, we need an ion Larmor radius correction, which however is ineffective when the ion density is flat, as in our last simulation. Note that an equilibrium potential $\varphi_{0}(y) \mathrm{im}-$ plies a radial electric field $E_{0 y}=-\varphi_{0}^{\prime}$ and a poloidal "rotation", since $V_{x}=V_{\| x}+\sin \theta E_{y} / B$. Our observation-that the presence of an equilibrium radial electric field and poloidal rotation can reduce the level of turbulence-may be relevant to plasma edge physics. Moreover, we shall demonstrate in the following section that transport across the magnetic field and stochastic heating are enhanced in the more turbulent $\left(\varphi_{0}=0\right)$ regimes.

\section{TEST PARTICLES AND TRANSPORT COEFFICIENTS}

One of the main objectives of plasma theory is the calculation of transport coefficients in magnetized plasmas. ${ }^{5-13}$ However, in spite of the abundance of theoretical models for "anomalous" transport in tokamaks, a full understanding of this phenomenon is still lacking. Numerical simulations, although idealized, can assist in relating experiments to theoretical estimates. In our case, the high accuracy of Vlasov Eulerian codes enables us to compute some interesting quantities from the Vlasov simulation. In order to sample regions of the phase space, we make use of test particles. These particles are driven by the electric fields computed from the Vlasov simulation, although they do not contribute to the creation of such fields. The particles follow the characteristics of the Vlasov equation (1):

$$
\begin{aligned}
& \frac{d x}{d t}=\frac{E_{y}^{*} \sin \theta}{B}+V_{\|} \cos \theta, \\
& \frac{d y}{d t}=-\frac{E_{x}^{*} \sin \theta}{B}, \\
& \frac{d V_{\|}}{d t}=\frac{e}{m_{i}} E_{x}^{*} \cos \theta .
\end{aligned}
$$

Note that, in writing the above characteristics, we have neglected the polarization drift, while retaining the Larmor radius correction (represented, as usual, by a star over the electric field). An interesting property of Eqs. (28) is that they possess the following invariant of the motion:

$$
P_{z} \equiv m_{i} V_{\|} \sin \theta+e B y \cos \theta=\mathrm{const}
$$



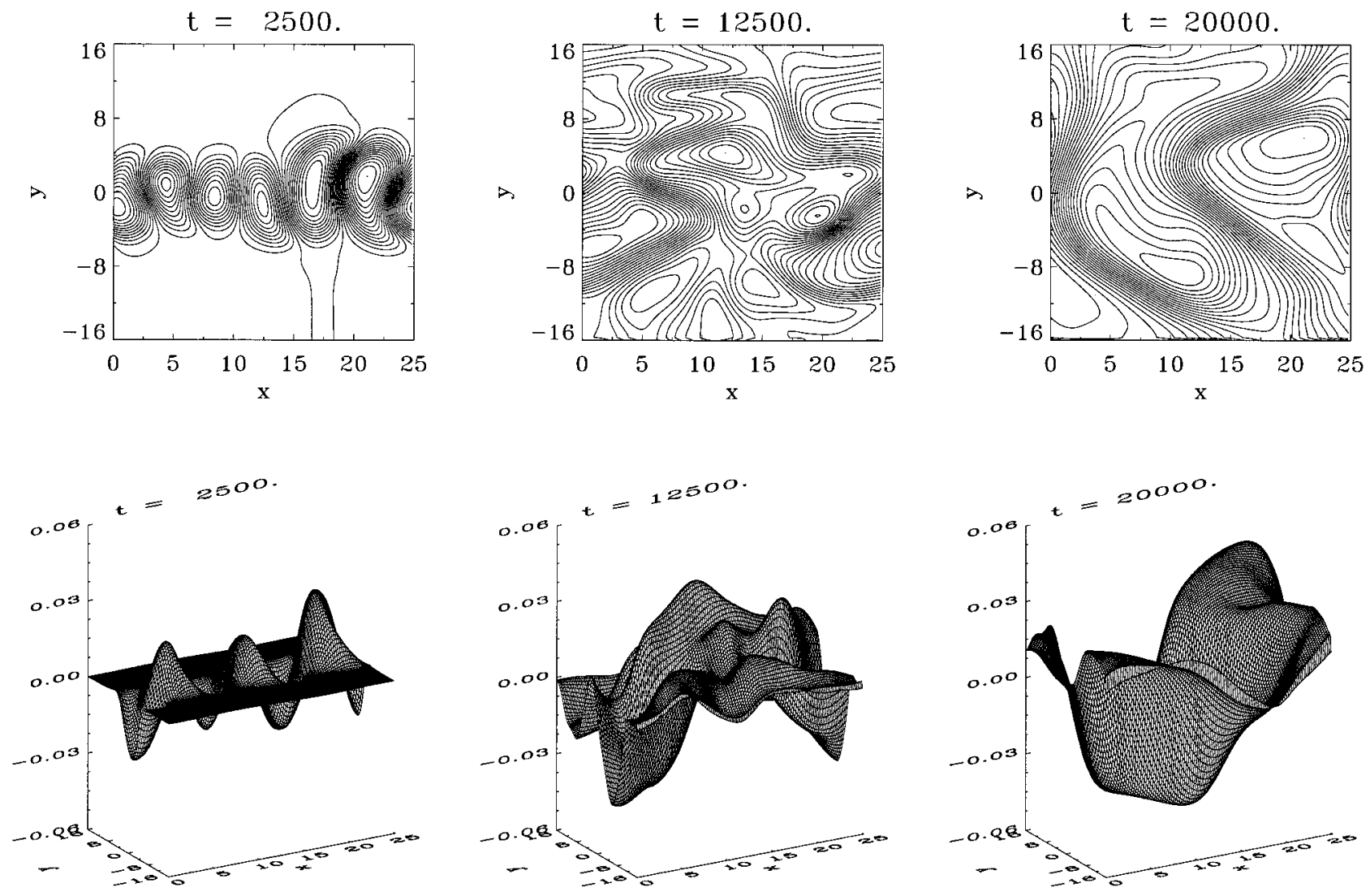

(a)

(b)

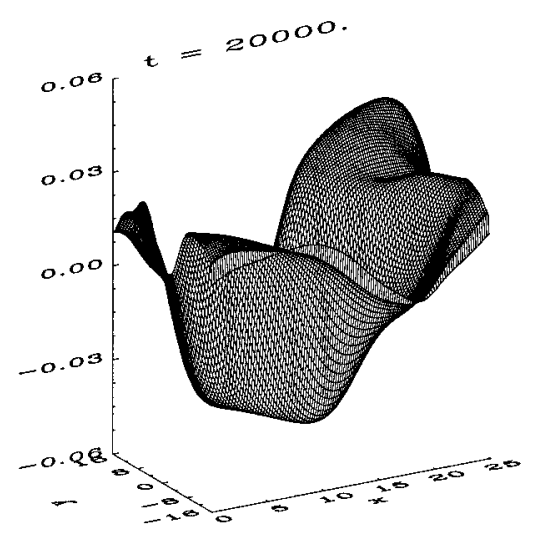

(c)

FIG. 14. Contour levels and three-dimensional view of the potential for the case of Fig. 13, at different times: $t=2500$ (a); $t=12500$ (b); $t=20000$ (c).

$P_{z}$ represents the $z$ component of the canonical momentum, which is of course conserved since $z$ is a cyclic coordinate. This invariant plays an important role in constraining particle diffusion in phase space.

We have followed the trajectories of 6000 test ions in three different cases. The particles are initialized after the saturation of the instability, in order to avoid the spurious growth of the transport coefficients driven by the instability itself. The particles are initially located at $-2<y<2$, $-0.5<V_{\|}<0.5$ and uniformly distributed in the $x$ direction. Diffusion coefficients are computed via the mean square displacements $\Delta y^{2}$ and $\Delta V_{\|}^{2}$, defined as

$$
\Delta y^{2}=\frac{1}{N_{\text {par }}} \sum_{i=1}^{N_{\text {par }}}\left[y_{i}(t)-y_{i}(0)\right]^{2} .
$$

A similar definition also holds for $\Delta V_{\|}^{2}$. These quantities are related to the diffusion coefficients $D_{y}$ and $D_{V_{\|}}$(respectively in space and velocity space) by

$$
D_{y}(t)=\frac{\Delta y^{2}}{t} ; \quad D_{V_{\|}}(t)=\frac{\Delta V_{\|}^{2}}{t} .
$$

The diffusion coefficients are plotted in Figs. 16(a) and 16(b), for two cases. The first case has already been investigated and has the following physical parameters: $L_{x}=25$, $\eta_{i}=6.1, \quad \nu=0, \quad L_{n}=14.1, \quad L_{T}=2.3, \quad \rho_{s}=\rho_{i}, T_{i 0}=T_{e}$ and $\theta=89.5^{\circ}$ (Fig. 16a). The second case (Fig. 16b) is the one we treated to illustrate the flat density profile, with $L_{x}=25$,
$L_{T}=10, \nu=10^{-4}, \rho_{i}=\rho_{s}, T_{i 0}=T_{e}, \theta=89.9^{\circ}$. Details for the evolution of the first case were previously described in Fig. 6, and for the second case in Fig. 13.

In both examples the diffusion coefficients grow quickly after initializing the particles, then adjust to an almost constant, or slowly decreasing, level. Note that the coefficients $D_{y}$ and $D_{V_{\|}}$are not independent, due to the invariant of Eq. (29), but must satisfy the relation

$$
\frac{D_{y}}{D_{V_{\|}}}=\frac{\tan ^{2} \theta}{\Omega_{i}^{2}} .
$$

This relation was accurately verified in the numerical calculation. The constraint imposed by Eq. (32) is important, because it predicts that diffusion in space and diffusion in velocity (heating) cannot occur independently. Of course, such a relation is no longer true when $k_{z} \neq 0$, but we can expect it to hold in an approximate way if the electric field $E_{z}$ stays small.

Concentrating our attention on $D_{y}$, we notice that, in the first case (Fig. 16a), $D_{y}$, after the initial transient, takes on a value $D_{y} \sim 10^{-3} \rho_{s}^{2} \Omega_{i}$. This low diffusion coefficient corresponds to a weakly turbulent regime. Turning to the second case (Fig. 16b), we find that now $D_{y} \sim 10^{-2} \rho_{s}^{2} \Omega_{i}$, i.e. an order of magnitude larger, and it remains almost perfectly constant after $\Omega_{i} t=16000$. The enhancement in the diffusion coefficient corresponds to the strongly turbulent regime previously observed in Fig. 14. 

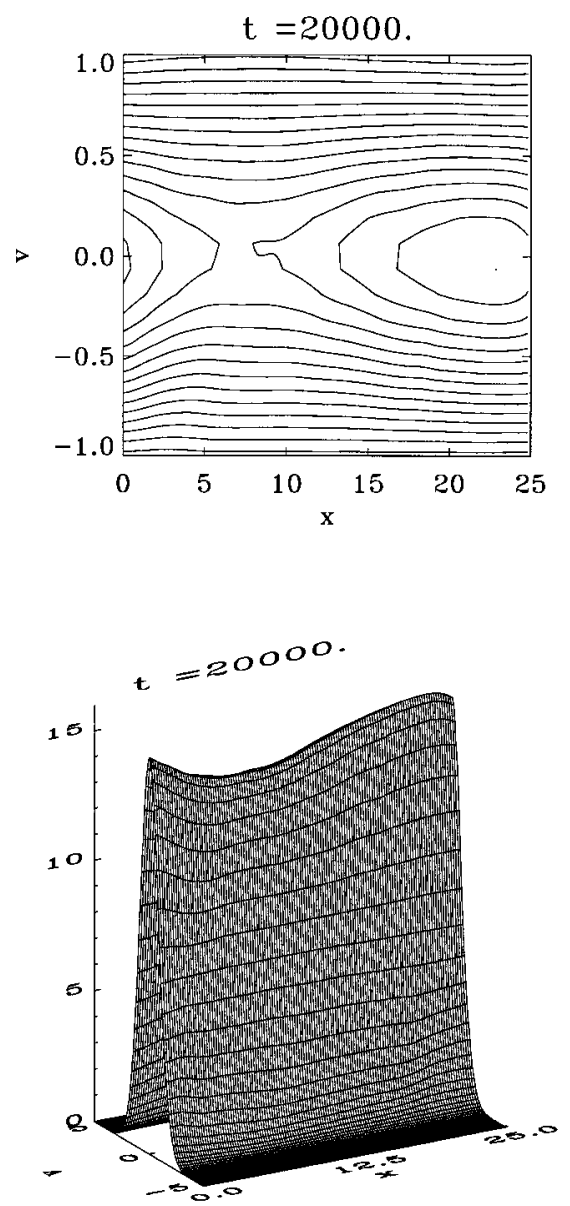

FIG. 15. Distribution function in the phase space $\left(x, V_{\|}\right)$for the same case as Fig. 13 at $t=20000$.

For tokamak-type plasma parameters $\left(T_{e} \approx 10 \mathrm{keV}\right.$ and $B \approx 5 \mathrm{~T}$ ), we have $\rho_{s}^{2} \Omega_{i} \approx 2 \times 10^{3} \mathrm{~m}^{2} \mathrm{~s}^{-1}$, giving, for our most strongly turbulent case:

$$
D_{y} \approx 20 \mathrm{~m}^{2} \mathrm{~s}^{-1} \text {. }
$$

This order of magnitude is broadly comparable with the values measured in tokamak experiments. ${ }^{11}$

\section{CONCLUSION}

We have presented a detailed numerical study of ITG instabilities in slab geometry using a Vlasov Eulerian code. Although ITG modes have attracted much attention during the last decade, virtually all simulations were performed with PIC codes. Our work is the first attempt to simulate ITG modes with an Eulerian code, which has the advantage of possessing a much lower level of noise than its PIC counterpart. This fact has enabled us to describe accurately the evolution of coherent structures, both in $(x, y)$ space and in phase space.

Our model takes into account the $\vec{E} \times \vec{B}$ and polarization drifts (with a correction for the ion Larmor radius), and is fully kinetic in the direction parallel to the magnetic field. It differs slightly from other models mainly in the treatment of the polarization drift. We have proven, however, that these models are essentially equivalent as far as long wavelengths are concerned. The electrons are treated as adiabatic, which has turned out to be a reasonable assumption since $\left|e \varphi / T_{e}\right| \ll 1$ in all our simulations.

The inverse cascade phenomenon was observed in the vast majority of our computer experiments. After a transient period, during which short wavelength structures may appear, the fundamental harmonic (which is imposed by the boundary conditions) becomes dominant. On expanding the boundaries, energy still flows to the larger scale structures that have become accessible.

As the angle $\theta$ between the magnetic field and the $x$ axis tends to $90^{\circ}$, modes with high wavenumbers become more unstable, since Landau damping is less and less effective. In the examples that we studied $\left(\eta_{i} \approx 6\right)$, we obtain stable behaviour for $\theta=85^{\circ}$, while for $\theta=89.5^{\circ}$ we observe a rapid initial growth of modes with $0.75<\rho_{s} k_{x}<1$. Trapped particles were observed in phase space, together with a nonnegligible deformation of the initially Maxwellian distribution function. These instabilities saturate by evolving towards a temperature profile with a smaller gradient, whereas the density profile shows little change.

It was found that the most strongly turbulent regimes are those in which either $\rho_{i} / \rho_{s}=\left(T_{i} / T_{e}\right)^{1 / 2} \ll 1$, or the equilibrium density profile is flat. Both these cases imply that the equilibrium potential $\varphi_{0}(y)$ is small (compared to $T_{e} / e$ ) or even zero. It could be argued that the presence of a finite equilibrium potential has the effect of reducing the turbulence level. Since $\varphi_{0}(y)$ is directly linked to the "radial" electric field $E_{0 y}$ and to the "poloidal" flow $V_{x}(y)$, this might provide an effective mechanism for turbulence suppression in the presence of a density gradient and a finite ion Larmor radius. We have also noticed that a small viscosity term in the ion Vlasov equation enhances the value of $E_{y}$.

The use of test particle techniques has confirmed that higher transport coefficients are to be expected in the most strongly turbulent regimes. We have observed diffusion coefficients as high as $D_{y} \sim 10^{-2} \rho_{s}^{2} \Omega_{i}$, which, for tokamaktype plasma parameters $\left(T_{e}=10 \mathrm{keV}, B=5 \mathrm{~T}\right)$, gives $D_{y} \sim 20$ $\mathrm{m}^{2} \mathrm{~s}^{-1}$. This result seems consistent with the suggestion that ITG modes can be responsible for the high diffusion coefficients observed in tokamak experiments. Note that so far we have considered only particle transport: energy transport is measured by the coefficient $D_{V_{\|}}$, but it turns out to be very low. This is simply due to the fact that we have taken into account only variations in the parallel ion temperature, while the perpendicular ion temperature is supposed to be uniform. Since the parallel component of the motion is very small, the diffusion coefficient $D_{V_{\|}}$is also small. A more meaningful study of energy transport would require a more complicated model, including gradients in the perpendicular temperature.

In summary, we have recovered many results previously obtained with PIC codes (essentially on global quantities such as growth rates or saturation levels). In addition, many more details about the evolution in phase space have been obtained, which have enabled us to determine the most strongly turbulent regimes. 

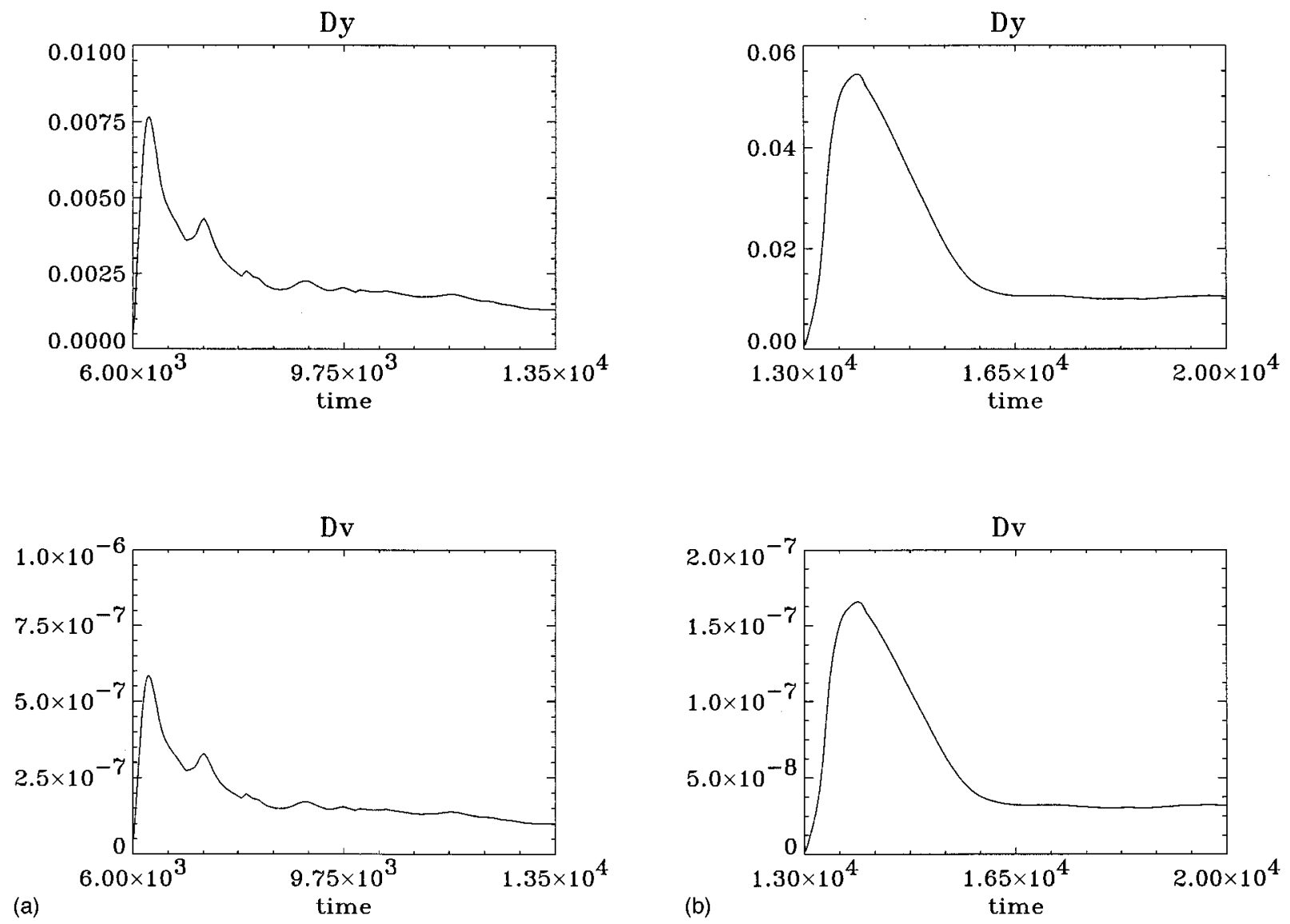

FIG. 16. Diffusion coefficients in real space (top frame) and velocity space (bottom frame) as a function of time. The parameters are: $L_{n}=14.1, \eta_{i}=6.1$, $\theta=89.5^{\circ}(\mathrm{a}) ; L_{n}=\eta_{i}=\infty, L_{T}=10, \theta=89.9^{\circ}$ (b).

\section{ACKNOWLEDGMENTS}

We acknowledge fruitful discussions with Dr. J. W. Connor, Dr. E. Fijalkow, Dr. M. R. Feix and Dr. M. Ottaviani.

This work was supported in part by the Commission of the European Communities under Contract No. ERBCHBICT941009 and was partially funded by the UK Department of Trade and Industry and Euratom.

\section{APPENDIX A: THE NUMERICAL VLASOV CODE}

The ion Vlasov equation (1a) can be rewritten in the following way:

$$
\begin{aligned}
& \frac{\partial F_{i}}{\partial t}+V_{x} \frac{\partial F_{i}}{\partial x}+V_{y} \frac{\partial F_{i}}{\partial y}+\frac{e}{m_{i}} E_{\|}^{*} \frac{\partial F_{i}}{\partial V_{\|}}+F_{i} \nabla_{\perp} \cdot \vec{V}_{p}=0, \\
& V_{x}=V_{E x}+V_{p x}+V_{\|} \sin \theta, \\
& V_{y}=V_{E y}+V_{p y} .
\end{aligned}
$$

Vlasov Eulerian codes are based on a uniform mesh on the entire phase space $\left(x, y, V_{\|}\right)$. Equation (A1) is solved by means of a splitting technique, which separately advances the distribution function in the $x, y$ and $V_{\|}$directions ${ }^{12-14}$. In order to advance $F_{i}$ by one time step, from $t$ to $t+\Delta t$, the following procedure is applied:

[STEP 1]: Solve, for a time $\Delta t / 2$, the equation

$$
\frac{\partial F_{i}}{\partial t}+V_{x} \frac{\partial F_{i}}{\partial x}=0
$$

which has the exact solution

$$
F_{i}^{+}\left(x, y, V_{\|}\right)=F_{i}\left(x-\frac{V_{x} \Delta t}{2}, y, V_{\|}, t\right) ;
$$

$F_{i}^{+}$is an intermediate result at an unspecified time between $t$ and $t+\Delta t$.

[STEP 2]: Solve for $\Delta t / 2$

$$
\begin{aligned}
& \frac{\partial F_{i}}{\partial t}+V_{y} \frac{\partial F_{i}}{\partial y}=0, \\
& F_{i}^{++}=F_{i}^{+}\left(x, y-\frac{V_{y} \Delta t}{2}, V_{\|}\right) .
\end{aligned}
$$

[STEP 3]: Solve for $\Delta t / 2$

$$
\begin{aligned}
& \frac{\partial F_{i}}{\partial t}+F_{i} \boldsymbol{\nabla} \cdot \vec{V}_{p}=0, \\
& F_{i}^{+++}=F_{i}^{++} \exp \left(-\frac{\Delta t}{2} \boldsymbol{\nabla} \cdot \vec{V}_{p}\right) .
\end{aligned}
$$

[STEP 4]: Compute the electrostatic potential from the quasineutrality relation: 


$$
\frac{e \varphi}{T_{e}}=\frac{n_{i}^{*}-n_{0}}{n_{0}}
$$

The electric fields are then calculated from $\varphi$ through the definition $\vec{E}=-\nabla \varphi$, and are subsequently smoothed using the filtering operator of Eq. (2) to obtain $\vec{E}^{*}$.

[STEP 5]: Repeat Step 3.

[STEP 6]: Repeat Step 2.

[STEP 7]: Repeat Step 1.

[STEP 8]: Solve for $\Delta t$ the following equation:

$$
\frac{\partial F_{i}}{\partial t}+\frac{e}{m_{i}} E_{\|}^{*} \frac{\partial F_{i}}{\partial V_{\|}}=0
$$

which gives the final result

$$
F_{i}\left(x, y, V_{\|}, t+\Delta t\right)=F_{i}\left(x, y, V_{\|}-\frac{e E_{\|}^{*}}{m_{i}} \Delta t\right),
$$

where the $F_{i}$ on the RHS of the previous equation is the result obtained after Step 7.

[STEP 9]: Repeat Step 4.

One then goes back to Step 1 and repeats the cycle. Note that the algorithm is symmetric, and centered around $t+\Delta t / 2$ (Step 4).

\section{APPENDIX B: ENERGY CONSERVATION}

We wish to prove, for the system of equations (1), (4) and (5), the existence of the invariant given in Eq. (6). We assume all quantities to be periodic in $x$, and $\varphi$ and $\nabla \varphi$ to vanish at $y= \pm L_{y}$. Let us begin by calculating the following (the subscript " $i$ " is understood for simplicity):

$$
\begin{aligned}
\frac{d}{d t} \frac{m}{2} \int F V_{\|}^{2} d \tau= & -\frac{m}{2} \int V_{\|}^{2} \nabla_{\perp} \cdot\left[\left(\vec{V}_{E}+\vec{V}_{p}\right) F\right] d \tau \\
& -\frac{e}{2} \int E_{\|}^{*} V_{\|}^{2} \frac{\partial F}{\partial V_{\|}} d \tau \\
& -\frac{m}{2} \int V_{\|}^{3} \nabla_{\|} F d \tau
\end{aligned}
$$

where $d \tau=d x d y d V_{\|}$. The first and the last of the terms on the RHS of Eq. (B1) vanish because of the boundary conditions. Thus, after some integrations by parts, we obtain

$$
\frac{d}{d t} \frac{m}{2} \int F V_{\|}^{2} d \tau=e \int V_{\|} \varphi^{*} \nabla_{\|} F d \tau .
$$

Now, we multiply the Eq. (1a) by $e \varphi^{*}$ and integrate in $d \tau$. We obtain

$$
\begin{aligned}
& \int e \varphi^{*} \frac{\partial F}{\partial t} d \tau+\int e \varphi^{*} \nabla_{\perp} \cdot\left(\vec{V}_{E} F\right) d \tau \\
& +\int e \varphi^{*} \nabla_{\perp} \cdot\left(\vec{V}_{p} F\right) d \tau-\frac{e^{2}}{m} \int \nabla_{\perp} \varphi^{*} \frac{\partial F}{\partial V_{\|}} d \tau \\
& +\int e \varphi^{*} V_{\|} \nabla_{\|} F d \tau=0 .
\end{aligned}
$$

The second and the fourth terms in the LHS of Eq. (B3) vanish. The first term can be written as follows

$$
\begin{aligned}
T_{e} \int \frac{e \varphi^{*}}{T_{e}} \frac{\partial F}{\partial t} d \tau & =\int T_{e} G *\left(\frac{n_{i}^{*}-n_{0}}{n_{0}}\right) \frac{\partial n_{i}}{\partial t} d x d y \\
& =T_{e} \int \frac{n_{i}^{*}-n_{0}}{n_{0}} \frac{\partial n_{i}^{*}}{\partial t} d x d y \\
& =T_{e} \frac{d}{d t} \int \frac{n_{i}^{* 2}}{2 n_{0}} d x d y \\
& =\frac{e}{2} \frac{d}{d t} \int n_{i}^{*} \varphi d x d y
\end{aligned}
$$

In deriving (B4) we have made use of Eqs. (2), (4) and (5). Now we consider the third term on the LHS of Eq. (B3).

$$
\begin{aligned}
e \int \varphi^{*} \nabla_{\perp} \cdot\left(\vec{V}_{p} F\right) d \tau & =e \int F \vec{V}_{p} \cdot E_{\perp}^{*} d \tau \\
& =\frac{e}{\Omega_{i} B} \int F \frac{d}{d t} E_{\perp}^{* 2} d \tau \\
& =\frac{e}{\Omega_{i} B}\left\{\int F \frac{\partial}{\partial t} \frac{E_{\perp}^{* 2}}{2} d \tau\right. \\
& \left.+\int F \vec{u} \cdot \nabla \frac{E_{\perp}^{* 2}}{2} d \tau\right\},
\end{aligned}
$$

where $\vec{u} \equiv \vec{V}_{E}+\vec{V}_{p}+\vec{V}_{\|}$. We use the identity $\nabla \cdot(a \vec{w})$ $=a \boldsymbol{\nabla} \cdot \vec{w}+\vec{w} \cdot \boldsymbol{\nabla} a$ in order to simplify the last term in Eq. (B5). We obtain

$$
\begin{aligned}
e \int & \varphi^{*} \nabla_{\perp} \cdot\left(\vec{V}_{p} F\right) d \tau \\
= & \frac{e}{\Omega_{i} B} \int F \frac{\partial}{\partial t} \frac{E_{\perp}^{* 2}}{2} d \tau-\frac{e}{\Omega_{i} B} \int \frac{E_{\perp}^{* 2}}{2} \nabla \cdot(\vec{u} F) d \tau \\
& =\frac{e}{2 \Omega_{i} B}\left\{\int F \frac{\partial E_{\perp}^{* 2}}{\partial t} d \tau+\int E_{\perp}^{* 2}\left[\frac{\partial F}{\partial t}+\frac{e}{m} E_{\|}^{*} \frac{\partial F}{\partial V_{\|}}\right] d \tau\right\} \\
& =\frac{e}{2 \Omega_{i} B} \frac{d}{d t} \int F E_{\perp}^{* 2} d \tau .
\end{aligned}
$$

In deriving Eq. (B6) we have made use of the Vlasov equation (1a). Moreover, the term in $\partial F / \partial V_{\|}$disappears after integration, since $F \rightarrow 0$ for $V_{\|} \rightarrow \pm \infty$. Returning to the Eq. (B3), we have now found that

$$
\begin{aligned}
\int e \varphi^{*} V_{\|} \boldsymbol{\nabla}_{\|} F d \tau= & -\frac{e}{2} \frac{d}{d t} \int n_{i}^{*} \varphi d x d y \\
& -\frac{e}{2 \Omega_{i} B} \frac{d}{d t} \int n_{i} E_{\perp}^{* 2} d x d y .
\end{aligned}
$$

Together with Eq. (B2), this provides us with the desired invariant

$$
\begin{gathered}
W=\frac{m}{2} \int F V_{\|}^{2} d \tau+\frac{e}{2} \int n_{i}^{*} \varphi d x d y \\
+\frac{m}{2 B^{2}} \int n_{i} E_{\perp}^{* 2} d x d y .
\end{gathered}
$$


${ }^{1}$ B. B. Kadomtsev and O. P. Pogutse, in Reviews of Plasma Physics (Consultants Bureau, New York, 1970), Vol. 5, p. 249.

${ }^{2}$ G. W. Hammett and F. W. Perkins, Phys. Rev. Lett. 64, 3019 (1990).

${ }^{3}$ G. W. Hammett, W. Dorland, and F. W. Perkins, Phys. Fluids B 4, 2052 (1992).

${ }^{4}$ W. Dorland and G. W. Hammett, Phys. Fluids B 5, 812 (1993).

${ }^{5}$ J. W. Connor and H. R. Wilson, Plasma Phys. Controlled Fusion 36, 719 (1994).

${ }^{6}$ J. W. Connor, Nucl. Fusion 26, 193 (1986).

${ }^{7}$ S. Hamaguchi and W. Horton, Phys Fluids B 2, 1833 (1990).

${ }^{8}$ A. B. Hassam, T. M. Antonsen, Jr., J. F. Drake, and P. N. Gudzar, Phys. Fluids B 2, 1822 (1990).

${ }^{9}$ G. S. Lee and P. H. Diamond, Phys. Fluids 29, 3291 (1986).

${ }^{10}$ P. W. Terry, J. N. Leboeuf, P. H. Diamond, D. R. Thayer, J. E. Sedlak, and G. S. Lee, Phys. Fluids 31, 2920 (1988).

${ }^{11}$ J. W. Connor, G. P. Maddison, H. R. Wilson, G. Corrigan, T. E. Stringer, and F. Tibone, Plasma Phys. Controlled Fusion 35, 319 (1993).

${ }^{12}$ S. E. Parker, W. Dorland, R. A. Santoro, M. Beer, Q. P. Liu, W. W. Lee, and G. W. Hammett, Phys. Plasmas 1, 1461 (1994).

${ }^{13}$ A. M. Dimits and W. W. Lee, Phys. Fluids B 3, 1557 (1991).
${ }^{14}$ M. Shoucri and R. Gagné, J Comput. Phys. 27, 315 (1978).

${ }^{15}$ A. Ghizzo, P. Bertrand, M. Shoucri, E. Fijalkow, M. R. Feix, J. Comput. Phys. 108, 105 (1993).

${ }^{16}$ G. Manfredi, M. Shoucri, M. R. Feix, P. Bertrand, E. Fijalkow, and A. Ghizzo, J. Comput. Phys. 121, 298 (1995).

${ }^{17}$ W. W. Lee and W. M. Tang, Phys. Fluids 31, 612 (1988).

${ }^{18}$ W. W. Lee, W. M. Tang, and H. Okuda, Phys. Fluids 23, 2007 (1980).

${ }^{19}$ B. I. Cohen, T. J. Williams, A. M. Dimits, and J. A. Byers, Phys. Fluids B 5, 2967 (1993).

${ }^{20}$ W. W. Lee, Phys. Fluids 26, 556 (1983).

${ }^{21}$ D. H. E. Dubin, J. A. Krommes, C. Oberman, and W. W. Lee, Phys. Fluids 26, 3524 (1983).

${ }^{22}$ G. Knorr, F. R. Hansen, J. P. Lynov, H. L. Pécseli, and J. J. Rasmussen, Phys. Scr. 38, 829 (1988).

${ }^{23}$ G. Knorr and H. Pécseli, J. Plasma Phys. 41, 157 (1989).

${ }^{24} \mathrm{D}$. Biskamp, in Turbulence and Anomalous Transport in Magnetized Plasmas, edited by D. Gresillon and M. A. Dubois (Editions de Physique, Orsay, 1987), p. 239.

${ }^{25}$ X. P. Huang, K. S. Fine, and C. F. Driscoll, Phys. Rev. Lett. 74, 4424 (1995). 